\title{
Asymptotic analysis of the high frequencies for the Laplace operator in a thin T-like shaped structure
}

\author{
Antonio Gaudiello ${ }^{(1), \star}$, Delfina Gómez ${ }^{(2)}$, Maria-Eugenia Pérez-Martínez ${ }^{(3)}$ \\ (1) Dipartimento di Matematica e Applicazioni "Renato Caccioppoli", \\ Università degli Studi di Napoli Federico II, Napoli, Italia \\ (2) Departamento de Matemáticas, Estadística y Computación, \\ Universidad de Cantabria, Santander, Spain \\ (3) Departamento de Matemática Aplicada y Ciencias de la Computación, \\ Universidad de Cantabria, Santander, Spain \\ * Corresponding author: gaudiell@unina.it
}

\begin{abstract}
We consider a spectral problem for the Laplacian operator in a planar T-like shaped thin structure $\Omega_{\varepsilon}$, where $\varepsilon$ denotes the transversal thickness of both branches. We assume the homogeneous Dirichlet boundary condition on the ends of the branches and the homogeneous Neumann boundary condition on the remaining part of the boundary of $\Omega_{\varepsilon}$. We study the asymptotic behavior, as $\varepsilon$ tends to zero, of the high frequencies of such a problem. Unlike the asymptotic behavior of the low frequencies where the limit problem involves only longitudinal vibrations along each branch of the T-like shaped thin structure (i.e. $1 D$ limit spectral problems), we obtain a two dimensional limit spectral problem which allows us to capture other kinds of vibrations. We also give a characterization of the asymptotic form of the eigenfunctions originating these vibrations.
\end{abstract}

Résumé: On considère le problème spectral pour le Laplacien, dans une structure mince bidimensionnelle $\Omega_{\varepsilon}$ en forme de $\mathrm{T}$, où $\varepsilon$ désigne l'épaisseur des deux branches du $\mathrm{T}$. Les conditions aux limites sont du type Neumann homogène sur tout le bord sauf aux extrémités des branches où une condition de Dirichlet homogène est imposée. On étudie le comportement asymptotique des hautes fréquences lorsque $\varepsilon$ tend vers zéro. Contrairement au comportement asymptotique des basses fréquences, pour lesquelles le problème limite ne fait apparaitre que des vibrations longitudinales le long de chaque branche de la structure (c'est-à-dire, des problèmes spectraux limites $1 D$ ), on obtient à la limite un problème spectral bidimensionnel, qui nous permet de capter d'autres types de vibrations. On donne également une caractérisation de la forme asymptotique des fonctions propres qui sont à l'origine de ces vibrations.

Keywords: Laplace operator; spectral problem; high frequencies; thin structures; junctions.

MSC: 35P05; 74K30; 35J05; 35P20; 35P15. 


\section{Introduction}

In this paper we study the asymptotic behavior of a spectral problem for the Laplacian operator in a planar T-like shaped thin structure $\Omega_{\varepsilon}$ (see Figure 1), as the thickness $\varepsilon$ of both branches tends to zero. More precisely, let

$$
\Omega_{\varepsilon}=(\omega \times]-\varepsilon, 0[) \bigcup(\varepsilon \omega \times[0, d[)
$$

and

$$
\Gamma_{\varepsilon}=(\partial \omega \times]-\varepsilon, 0[) \bigcup(\varepsilon \omega \times\{d\})
$$

a part of its boundary, where $d \in] 0,+\infty[$ and $\omega \subset \mathbb{R}$ is an open bounded interval such that $0 \in \omega$.

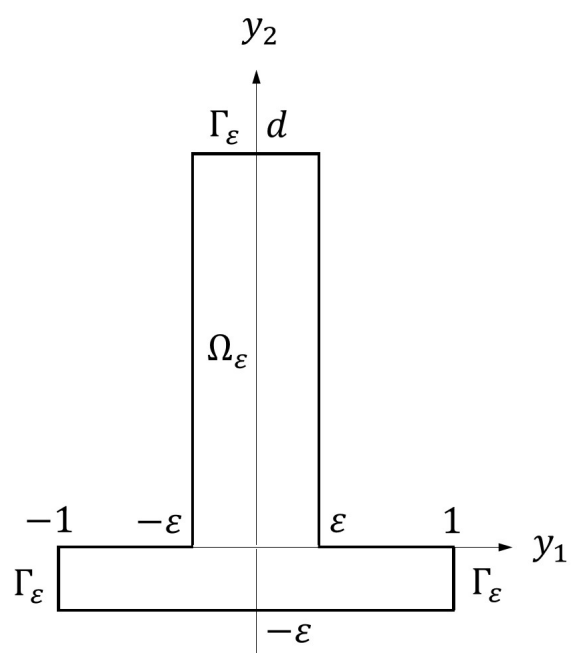

Figure 1: The T-like shaped structure $\Omega_{\varepsilon}$, when $\left.\omega=\right]-1,1[$

In $\Omega_{\varepsilon}$, we consider the following eigenvalue problem

$$
\left\{\begin{array}{l}
-\triangle U_{\varepsilon}=\lambda_{\varepsilon} U_{\varepsilon}, \text { in } \Omega_{\varepsilon}, \\
U_{\varepsilon}=0, \text { on } \Gamma_{\varepsilon}, \\
\frac{\partial U_{\varepsilon}}{\partial \nu}=0, \text { on } \partial \Omega_{\varepsilon} \backslash \Gamma_{\varepsilon}
\end{array}\right.
$$

where $\nu$ denotes the unit outer normal on $\partial \Omega_{\varepsilon}$. For each $\varepsilon$, let

$$
0<\lambda_{\varepsilon, 1} \leq \lambda_{\varepsilon, 2} \leq \cdots \leq \lambda_{\varepsilon, k} \leq \cdots \stackrel{k \rightarrow+\infty}{\longrightarrow}+\infty
$$

be the sequence of all the eigenvalues of (1.1), with the classical convention of repeated eigenvalues. The mini-max principle provides (see Section 2)

$$
\exists \lambda_{1}^{*}>0: \quad \lambda_{1}^{*} \leq \lambda_{\varepsilon, k} \leq \frac{k^{2} \pi^{2}}{d^{2}}, \quad \forall k \in \mathbb{N}, \quad \forall \varepsilon .
$$


This estimate asserts that the so-called low frequencies (i.e. the sequences $\left\{\lambda_{\varepsilon, k}\right\}_{\varepsilon}$, for any fixed $k$ ) are of order $O(1)$. Precisely, in [17] (see also [18]), as $\varepsilon$ tends to zero, the authors obtain a limit spectral problem (see (2.11)) composed by two $1 D$ differential equations whose solutions are coupled by a junction condition (see Theorem 2.1). This limit problem is posed on the skeleton of the $T$-like structure, namely for $\left(x_{1}, x_{2}\right) \in$ $(\omega \times\{0\}) \bigcup(\{0\} \times] 0, d[)$, while a Dirichlet condition is imposed on the extremes $\partial \omega$ and at $x_{2}=d$.

Nevertheless, as it happens in many singularly perturbed problems (see, for instance, [20], [21], [22], [31], [32], and [42]), there are sequences of eigenvalues $\left\{\lambda_{\varepsilon}=\lambda_{\varepsilon, k(\varepsilon)}\right\}_{\varepsilon}$ of order $O\left(\varepsilon^{-\gamma}\right)$ with $k(\varepsilon) \rightarrow \infty$ and for some $\gamma>0$, the so-called high frequencies, whose corresponding eigenfunctions $U_{\varepsilon}=U_{\varepsilon, k(\varepsilon)}$, suitably normalized, do not vanish asymptotically. The goal of this paper is to localize those sequences of eigenvalues giving rise to other kinds of vibrations, such as the transverse vibrations of the $T$-like shaped structure, and provide information on the structure of the corresponding eigenfunctions. It should be emphasized that this is closely linked with the normalization for the eigenfunctions of (1.1) that we choose (see (3.3)).

At first (see Section 2), problem (1.1) is reformulated on the fixed domain $\Omega^{a} \bigcup \Omega^{b}$, $\left.\Omega^{a}=\omega \times\right] 0, d\left[, \Omega^{b}=\omega \times\right]-1,0[$, through suitable rescalings (see (2.4)), and the asymptotic behavior of the low frequencies, as $\varepsilon$ tends to zero, is recalled. Then (see Section 3), we prove that $\varepsilon^{\gamma} \lambda_{\varepsilon}$, for $\gamma>0$ and $\lambda_{\varepsilon}$ eigenvalue of (1.1), accumulate along all the positive real axis (see Proposition 3.2 and Remark 3.3). However, only for the eigenvalues $\lambda_{\varepsilon}$ of (1.1) such that $\varepsilon^{2} \lambda_{\varepsilon}$ are asymptotically near an eigenvalue of one of the following $1 D$ Neumann spectral problems

$$
\left\{\begin{array}{l}
-\left(z^{a}\right)^{\prime \prime}=\lambda^{a} z^{a}, \text { in } \omega \\
\left(z^{a}\right)^{\prime}=0, \text { on } \partial \omega
\end{array}\right.
$$

or

$$
\left\{\begin{array}{l}
\left.-\left(z^{b}\right)^{\prime \prime}=\lambda^{b} z^{b}, \text { in }\right]-1,0[, \\
\left(z^{b}\right)^{\prime}(-1)=0=\left(z^{b}\right)^{\prime}(0),
\end{array}\right.
$$

the associated eigenfunctions, normalized by $\left\|U_{\varepsilon}\right\|_{L^{2}\left(\Omega_{\varepsilon}\right)}^{2}=\varepsilon$, can be asymptotically nonzero (see Proposition 3.4, Corollary 3.5, Proposition 3.6, Proposition 4.1, and Proposition 4.2). Moreover, in Section 5, for any fixed eigenvalue $\lambda$ of (1.3) or (1.4), we prove that

$$
\forall \varepsilon, \quad \exists \lambda_{\varepsilon} \text { eigenvalue of }(1.1): \quad\left|\varepsilon^{2} \lambda_{\varepsilon}-\lambda\right| \leq c \varepsilon^{\frac{3}{2}},
$$

where $c$ is a constant independent of $\varepsilon$. However, obtaining (1.5) involves the introduction of a correcting term $\varepsilon^{2} \mu$, where the parameter $\mu$ is identified (see Proposition 4.5) with the eigenvalues of another two $1 D$ Dirichlet spectral problems in the skeleton of the structure, namely, of problems

$$
\left\{\begin{array}{l}
\left.-\alpha^{\prime \prime}=\mu \alpha, \text { in }\right] 0, d[, \\
\alpha(0)=\alpha(d)=0,
\end{array}\right.
$$


and

$$
\left\{\begin{array}{l}
-\beta^{\prime \prime}=\mu \beta, \text { in } \omega \backslash\{0\}, \\
\beta=0, \text { on } \partial \omega \cup\{0\},
\end{array}\right.
$$

Furthermore, roughly speaking, any fixed function of the type

$$
z^{a}\left(x_{1}\right) \alpha\left(x_{2}\right) \text { in } \Omega^{a} \text { and } \beta\left(x_{1}\right) z^{b}\left(x_{2}\right) \text { in } \Omega^{b},
$$

where $z^{a}$ and $z^{b}$ are eigenfunctions of (1.3) and (1.4), and $\alpha$ and $\beta$ are eigenfunctions of the problems (1.6) and (1.7), is limit of "groups" eigenfunctions associated with all the eigenvalues $\lambda_{\varepsilon}$ belonging to small intervals centered at $\lambda$ (see (1.5), Theorems 5.2, 5.3, and 5.4 for the precise statements). Section 4 is devoted to "guessing" the decomposition structure of functions in (1.8), while a complete characterization of the eigenfunctions associated with eigenvalues $\lambda_{\varepsilon}$ of (1.1) of order $O\left(\varepsilon^{-2}\right)$ is given in Theorem 5.6.

We explicitly notice that the low frequencies are related only with longitudinal vibrations along each branch of the T-like shaped thin structure (see Theorem 2.1 and Figure 2 (a)), while our results capture also vibrations depending on both variables, which are referred to as transverse vibrations along each branch, and are produced by high frequencies (see Theorems 5.2-5.4, Remark 5.5 and Figure $2(\mathrm{~b})$ ). In fact, we get results for eigenvalues and eigenfunctions of (1.1) which are of interest in terms of the associated evolution problems since, from (1.8), we can construct standing waves which approach time-dependent solutions for long times, and these times can be precisely computed in terms of bounds for discrepancies such as that in (1.5) (see [41] and [34] for an abstract framework as well as for applications to very different vibrating systems).

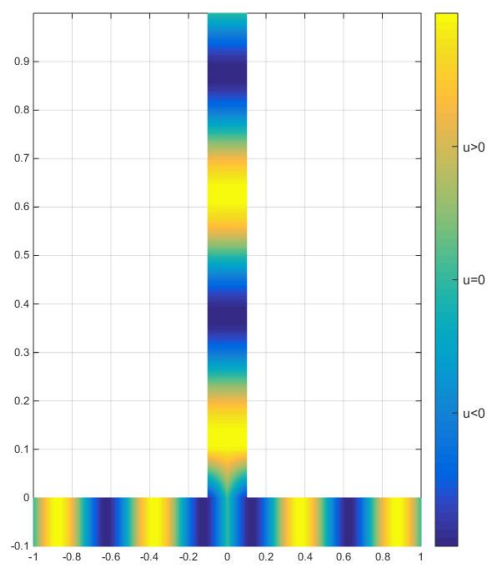

(a)

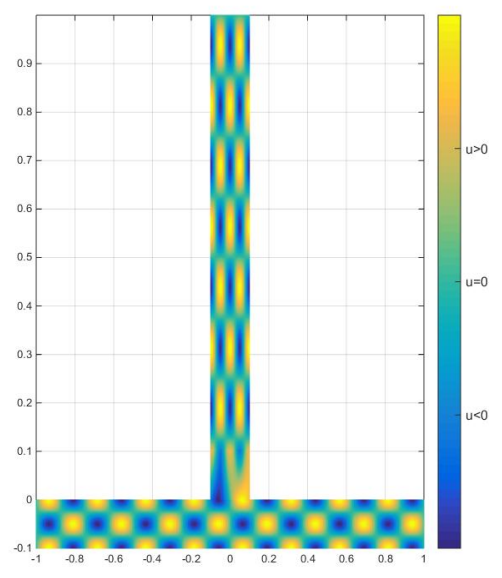

(b)

Figure 2: Examples of approximation of eigenfunctions giving rise to longitudinal and transverse vibrations for $\omega=]-1,1[$ and $d=1$. Figure (a) is obtained by choosing the eigenvalue $\mu=16 \pi^{2}$ in (2.11). Figure (b) is obtained using formula (5.19) with $\lambda^{a}=\lambda^{b}=4 \pi^{2}, \mu^{a}=\mu^{b}=64 \pi^{2}$, and $\varepsilon=0.1$.

It should be emphasized that it seems to be a common fact to many mechanical systems arising in thin structures that the low frequencies give rise to longitudinal vibrations while 
for other kinds of vibrations such as torsional or stretching vibrations one must look among those associated to the high frequencies: see, for instance, [8], [23], [24], [35] and references therein. Also, we emphasize that, to our knowledge, this is the first work in the literature addressing the asymptotics of the high frequencies for a thin T-like shaped structure. About the study of spectral problems for the Laplace operator in (bounded or unbounded) thin trips and tubes we refer to [1], [2], [3], [5], [10], [11], [29], and [39]. Some of these references also address the phenomena of the localization of certain kinds of vibrations concentrating asymptotically its support in regions where the geometry of the problem has some kind of perturbation. Related to the localization of eigenfunctions giving rise to vibrations concentrated at points or along certain regions of the structure we mention [22], [25], [33], [36], [40], [42]. About the study of junctions as considered in this paper but in other contexts, we refer to [4], [9], [12], [14], [15], [16], [19], [26], [27], [28], [30] and [37].

Finally, we summarize the structure of the paper as follows. Section 2 contains the statement of the problem, classical techniques from $T$-like shaped structures and some preliminary results on the low frequencies. Section 3 contains some results for the high frequencies which among other things provide a limit spectral problem and a critical parameter for which the associated eigenfunctions are asymptotically different from zero in the weak topology of $L^{2}\left(\Omega^{a} \bigcup \Omega^{b}\right)$. Section 4 contains an analysis of the limit spectral problem and a corrector term which is essential to detect the asympotic form of the above mentioned eigenfunctions. Finally, Section 5 contains the main results of the paper.

\section{Statement of the problem and some results on low frequencies}

In what follows, both $y=\left(y_{1}, y_{2}\right)$ and $x=\left(x_{1}, x_{2}\right)$ denote a generic element of $\mathbb{R}^{2},|\omega|$ denotes the length of interval $\omega$, and $\varepsilon \in] 0, \min \{1, d\}[$.

The weak formulation of (1.1) is

$$
\left\{\begin{array}{l}
U_{\varepsilon} \in \mathcal{V}^{\varepsilon}, \quad U_{\varepsilon} \neq 0 \\
\int_{\Omega_{\varepsilon}} D U_{\varepsilon} D V d y=\lambda_{\varepsilon} \int_{\Omega_{\varepsilon}} U_{\varepsilon} V d y, \quad \forall V \in \mathcal{V}^{\varepsilon},
\end{array}\right.
$$

where $\mathrm{D}$ stands for the gradient and

$$
\mathcal{V}_{\varepsilon}=\left\{V \in H^{1}\left(\Omega_{\varepsilon}\right): V=0 \text { on } \Gamma_{\varepsilon}\right\}
$$

Let us set

$$
\left\{\begin{array}{l}
\left.\Omega^{a}=\omega \times\right] 0, d\left[, \quad \Omega^{b}=\omega \times\right]-1,0[, \\
\left.\Gamma^{a}=\omega \times\{d\}, \quad \Gamma^{b}=\partial \omega \times\right]-1,0[.
\end{array}\right.
$$

As it is usual (see [7]), problem (2.1) is reformulated on the fixed domain $\Omega^{a} \cup \Omega^{b}$ through the maps

$$
\left\{\begin{array}{l}
\left.x=\left(x_{1}, x_{2}\right) \in \Omega^{a} \longrightarrow\left(\varepsilon x_{1}, x_{2}\right) \in \Omega_{\varepsilon}^{a}=\varepsilon \omega \times\right] 0, d[ \\
\left.x=\left(x_{1}, x_{2}\right) \in \Omega^{b} \longrightarrow\left(x_{1}, \varepsilon x_{2}\right) \in \Omega_{\varepsilon}^{b}=\omega \times\right]-\varepsilon, 0[
\end{array}\right.
$$


Precisely, if $\left(\lambda_{\varepsilon}, U_{\varepsilon}\right)$ satisfies $(2.1)$ then $\left(\lambda_{\varepsilon}, u_{\varepsilon}\right)$, with $u_{\varepsilon}=\left(u_{\varepsilon}^{a}, u_{\varepsilon}^{b}\right)$ defined by

$$
u_{\varepsilon}^{a}\left(x_{1}, x_{2}\right)=U_{\varepsilon}\left(\varepsilon x_{1}, x_{2}\right) \text { in } \Omega^{a}, \quad u_{\varepsilon}^{b}\left(x_{1}, x_{2}\right)=U_{\varepsilon}\left(x_{1}, \varepsilon x_{2}\right) \text { in } \Omega^{b},
$$

satisfies

$$
\left\{\begin{array}{l}
u_{\varepsilon}=\left(u_{\varepsilon}^{a}, u_{\varepsilon}^{b}\right) \in V_{\varepsilon}, \quad u_{\varepsilon} \not \equiv 0, \\
\int_{\Omega^{a}}\left(\partial_{x_{1}} u_{\varepsilon}^{a} \partial_{x_{1}} v^{a}+\varepsilon^{2} \partial_{x_{2}} u_{\varepsilon}^{a} \partial_{x_{2}} v^{a}\right) d x+\int_{\Omega^{b}}\left(\varepsilon^{2} \partial_{x_{1}} u_{\varepsilon}^{b} \partial_{x_{1}} v^{b}+\partial_{x_{2}} u_{\varepsilon}^{b} \partial_{x_{2}} v^{b}\right) d x \\
=\varepsilon^{2} \lambda_{\varepsilon}\left(\int_{\Omega^{a}} u_{\varepsilon}^{a} v^{a} d x+\int_{\Omega^{b}} u_{\varepsilon}^{b} v^{b} d x\right), \quad \forall\left(v^{a}, v^{b}\right) \in V_{\varepsilon},
\end{array}\right.
$$

where

$$
\begin{aligned}
& V_{\varepsilon}=\left\{v=\left(v^{a}, v^{b}\right) \in H^{1}\left(\Omega^{a}\right) \times H^{1}\left(\Omega^{b}\right): v^{a}\left(x_{1}, 0\right)=v^{b}\left(\varepsilon x_{1}, 0\right) \text { on } \omega,\right. \\
& \left.v^{a}=0 \text { on } \Gamma^{a}, \quad v^{b}=0 \text { on } \Gamma^{b}\right\} .
\end{aligned}
$$

Conversely, if $\left(\lambda_{\varepsilon}, u_{\varepsilon}\right)$ satisfies $(2.6)$ then $\left(\lambda_{\varepsilon}, U_{\varepsilon}\right)$, with $U_{\varepsilon}$ defined by

$$
U_{\varepsilon}\left(y_{1}, y_{2}\right)=u_{\varepsilon}^{a}\left(\frac{y_{1}}{\varepsilon}, y_{2}\right) \text { in } \Omega_{\varepsilon}^{a}, \quad U_{\varepsilon}\left(y_{1}, y_{2}\right)=u_{\varepsilon}^{b}\left(y_{1}, \frac{y_{2}}{\varepsilon}\right) \text { in } \Omega_{\varepsilon}^{b},
$$

satisfies (2.1).

The mini-max principle allows us to obtain estimate (1.2) with $\lambda_{1}^{*}$ denoting the first eigenvalue of the following problem

$$
\left\{\begin{array}{l}
w=\left(w^{a}, w^{b}\right) \in V^{*} \\
\int_{\Omega^{a}} D w^{a} D v^{a} d x+\int_{\Omega^{b}} D w^{b} D v^{b} d x \\
=\lambda^{*}\left(\int_{\Omega^{a}} w^{a} v^{a} d x+\int_{\Omega^{b}} w^{b} v^{b} d x\right), \quad \forall\left(v^{a}, v^{b}\right) \in V^{*},
\end{array}\right.
$$

where

$$
V^{*}=\left\{v=\left(v^{a}, v^{b}\right) \in H^{1}\left(\Omega^{a}\right) \times H^{1}\left(\Omega^{b}\right): v^{a}=0 \text { on } \Gamma^{a}, \quad v^{b}=0 \text { on } \Gamma^{b}\right\} .
$$

As far as the proof of the upper bound is concerned, we refer to [17]. To prove the lower bound, we use the characterization of the first eigenvalue of (2.1) and of (2.8). Indeed,

$$
\lambda_{\varepsilon, 1}=\min _{V \in \mathcal{V}_{\varepsilon} \backslash\{0\}} \frac{\int_{\Omega_{\varepsilon}}|D V|^{2} d y}{\int_{\Omega_{\varepsilon}}|V|^{2} d y} \geq \min _{\left(v^{a}, v^{b}\right) \in V^{*} \backslash\{0\}} \frac{\int_{\Omega^{a}}\left|D v^{a}\right|^{2} d x+\int_{\Omega^{b}}\left|D v^{b}\right|^{2} d x}{\int_{\Omega^{a}}\left|v^{a}\right|^{2} d x+\int_{\Omega^{b}}\left|v^{b}\right|^{2} d x}=\lambda_{1}^{*} .
$$

Estimates (1.2) allow us to assert that the so-called low frequencies are of order $O(1)$. Namely, we recall the following result proved in [17]. 
Theorem 2.1. For each $\varepsilon \in] 0, \min \{1, d\}\left[\right.$, let $\left\{\lambda_{\varepsilon, k}\right\}_{k \in \mathbb{N}}$ be the sequence of all the eigenvalues of (2.1). Let

$$
0<\mu_{1} \leq \mu_{2} \leq \cdots \leq \mu_{k} \leq \cdots \stackrel{k \rightarrow+\infty}{\longrightarrow}+\infty,
$$

be the sequence of all the eigenvalues (with the classical convention of repeated eigenvalues) of

$$
\left\{\begin{array}{l}
\left(u^{a}, u^{b}\right) \in \mathcal{V}, \\
|\omega| \int_{0}^{d}\left(u^{a}\right)^{\prime}\left(v^{a}\right)^{\prime} d y_{2}+\int_{\omega}\left(u^{b}\right)^{\prime}\left(v^{b}\right)^{\prime} d y_{1} \\
=\mu\left(|\omega| \int_{0}^{d} u^{a} v^{a} d y_{2}+\int_{\omega} u^{b} v^{b} d y_{1}\right), \quad \forall\left(v^{a}, v^{b}\right) \in \mathcal{V},
\end{array}\right.
$$

where

$$
\begin{aligned}
\mathcal{V}= & \left\{v=\left(v^{a}, v^{b}\right) \in H^{1}(] 0, d[) \times H^{1}(\omega): v^{a}(d)=0, v^{b}=0 \text { on } \partial \omega,\right. \\
& \left.v^{a}(0)=v^{b}(0)\right\} .
\end{aligned}
$$

Then,

$$
\lim _{\varepsilon \rightarrow 0} \lambda_{\varepsilon, k}=\mu_{k}, \quad \forall k \in \mathbb{N} .
$$

Let $\{\varepsilon\} \subset] 0, \min \{1, d\}[$ be a sequence tending to zero and, for each $\varepsilon$ in such a sequence, let $\left\{\left(\lambda_{\varepsilon, k}, U_{\varepsilon, k}\right)\right\}_{k \in \mathbb{N}}$ be a sequence of eigenelements of (2.1) with $\left\{U_{\varepsilon, k}\right\}_{k \in \mathbb{N}}$ an orthonormal basis in $L^{2}\left(\Omega_{\varepsilon}\right)$. Then, there exists a subsequence of $\{\varepsilon\}$, still denoted by $\{\varepsilon\}$, and a sequence $\left\{\left(u_{k}^{a}, u_{k}^{b}\right)\right\}_{k \in \mathbb{N}} \subset \mathcal{V}$ such that

$$
\begin{aligned}
& \lim _{\varepsilon \rightarrow 0} \int_{\varepsilon \omega \times] 0, d[}\left(\left|U_{\varepsilon, k}-\varepsilon^{-\frac{1}{2}} u_{k}^{a}\right|^{2}+\left|\partial_{y_{1}} U_{\varepsilon, k}\right|^{2}+\left|\partial_{y_{2}} U_{\varepsilon, k}-\varepsilon^{-\frac{1}{2}}\left(u_{k}^{a}\right)^{\prime}\right|^{2}\right) d y=0, \forall k \in \mathbb{N}, \\
& \lim _{\varepsilon \rightarrow 0} \int_{\omega \times]-\varepsilon, 0[}\left(\left|U_{\varepsilon, k}-\varepsilon^{-\frac{1}{2}} u_{k}^{b}\right|^{2}+\left|\partial_{y_{1}} U_{\varepsilon, k}-\varepsilon^{-\frac{1}{2}}\left(u_{k}^{b}\right)^{\prime}\right|^{2}+\left|\partial_{y_{2}} U_{\varepsilon, k}\right|^{2}\right) d y=0, \forall k \in \mathbb{N},
\end{aligned}
$$

and $u_{k}=\left(u_{k}^{a}, u_{k}^{b}\right)$ is an eigenfunction of problem (2.9) with eigenvalue $\mu_{k}$. Furthermore, $\left\{u_{k}\right\}_{k \in \mathbb{N}}$ is an orthonormal basis in $L^{2}(] 0, d[) \times L^{2}(\omega)$ with the inner product

$$
(u, v)=|\omega| \int_{0}^{d} u^{a} v^{a} d y_{2}+\int_{\omega} u^{b} v^{b} d y_{1}, \quad \forall u, v \in \mathcal{V} .
$$


Remark that (2.9) is the weak formulation of

$$
\left\{\begin{array}{l}
\left.-\left(u^{a}\right)^{\prime \prime}=\mu u^{a}, \text { in }\right] 0, d[ \\
-\left(u^{b}\right)^{\prime \prime}=\mu u^{b}, \text { in } \omega \backslash\{0\}, \\
u^{a}(d)=0, \\
u^{b}=0, \text { on } \partial \omega, \\
u^{a}(0)=u^{b}(0), \\
|\omega|\left(u^{a}\right)^{\prime}(0)=\left(\left(u^{b}\right)^{\prime}\left(0^{-}\right)-\left(u^{b}\right)^{\prime}\left(0^{+}\right)\right),
\end{array}\right.
$$

where $u^{\prime}$ and $u^{\prime \prime}$ denote the first and the second derivative of $u$, respectively.

\section{A critical order for high frequencies}

In this section we study the asymptotic behavior of the eigenvalues $\lambda_{\varepsilon}$ of (2.1) of order of magnitude $O\left(\varepsilon^{-\gamma}\right)$ for $\gamma>0$ and the asymptotic behavior of the corresponding eigenfunctions (the case $\gamma=0$ is recalled in Theorem 2.1). We prove that $\varepsilon^{\gamma} \lambda_{\varepsilon}$ accumulate along all the positive real axis for any $\gamma>0$ (see Proposition 3.2 and Remark 3.3). Moreover, we prove that there exists a different behavior of the corresponding eigenfunctions depending on whether $\varepsilon^{2} \lambda_{\varepsilon}$ are asymptotically near an eigenvalue of (3.7) or not (see Proposition 3.4, Corollary 3.5, and Proposition 3.6). As a matter of fact, on the basis of the normalization (3.4) and the convergence (3.13), only when $\gamma=2$ and $\lambda$ is an eigenvalue of the limit problem (3.7) the associated eigenfunctions can converge towards some function different from zero.

At first, we recall the following result proved in [6].

Theorem 3.1. Let $\left\{\mathcal{T}_{\varepsilon}\right\}_{\varepsilon \in[0,1]}$ be a family of selfadjoint and compact operators on a Hilbert space $H$. For each $\varepsilon \in[0,1]$, let $\left\{\mu_{\varepsilon, k}\right\}_{k \in \mathbb{N}}$ be the sequence of all the eigenvalues of $\mathcal{T}_{\varepsilon}$ with the classical convention of repeated eigenvalues. Assume that the family $\left\{\mathcal{T}_{\varepsilon}\right\}_{\varepsilon \in[0,1]}$ satisfies the following property:

$$
\forall k \in \mathbb{N} \text {, the function } \varepsilon \in[0,1] \rightarrow \mu_{\varepsilon, k} \text { is continuous. }
$$

Then, for each $\gamma \in] 0,+\infty[$ and $\lambda \in] 0,+\infty\left[\right.$ there exists a vanishing sequence $\left\{\varepsilon_{j}\right\}_{j \in \mathbb{N}} \subset$ $[0,1]$ and a diverging sequence of integer positive numbers $\left\{k\left(\varepsilon_{j}\right)\right\}_{j \in \mathbb{N}}$ such that

$$
\varepsilon_{j}^{\gamma}\left(\mu_{\varepsilon_{j}, k\left(\varepsilon_{j}\right)}\right)^{-1}=\lambda \text {. }
$$

Proposition 3.2. For each $\varepsilon \in] 0, \min \{1, d\}\left[\right.$, let $\left\{\lambda_{\varepsilon, k}\right\}_{k \in \mathbb{N}}$ be the sequence of all the eigenvalues of (2.1). Then, for each $\gamma \in] 0,+\infty[$ and $\lambda \in] 0,+\infty[$, there exists a vanishing sequence $\left\{\varepsilon_{j}\right\}_{j \in \mathbb{N}}$ and a diverging sequence of positive integer numbers $\left\{k\left(\varepsilon_{j}\right)\right\}_{j \in \mathbb{N}}$ such that

$$
\varepsilon_{j}^{\gamma} \lambda_{\varepsilon_{j}, k\left(\varepsilon_{j}\right)}=\lambda
$$


Proof. Let $\left\{\mathcal{T}_{\varepsilon}\right\}_{\varepsilon \in[0,1]}$ be the family of operators defined by

$$
\mathcal{T}_{0}: w=\left(w^{a}, w^{b}\right) \in \mathcal{V} \longrightarrow \mathcal{T}_{0}(w) \in \mathcal{V}
$$

where

$$
\left\{\begin{array}{l}
\mathcal{T}_{0}(w)=\left(\mathcal{T}_{0}(w)^{a}, \mathcal{T}_{0}(w)^{b}\right) \in \mathcal{V} \\
|\omega| \int_{0}^{d} \partial_{y_{2}} \mathcal{T}_{0}(w)^{a} \partial_{y_{2}} v^{a} d y_{2}+\int_{\omega} \partial_{y_{1}} \mathcal{T}_{0}(w)^{b} \partial_{y_{1}} v^{b} d y_{1} \\
=|\omega| \int_{0}^{d} w^{a} v^{a} d y_{2}+\int_{\omega} w^{b} v^{b} d y_{1}, \quad \forall\left(v^{a}, v^{b}\right) \in \mathcal{V}
\end{array}\right.
$$

with $\mathcal{V}$ defined in $(2.10)$, if $\varepsilon=0$;

$$
\mathcal{T}_{\varepsilon}: W \in \mathcal{V}_{\varepsilon} \longrightarrow \mathcal{T}_{\varepsilon}(W) \in \mathcal{V}_{\varepsilon}
$$

where

$$
\left\{\begin{array}{l}
\mathcal{T}_{\varepsilon}(W) \in \mathcal{V}_{\varepsilon}, \\
\int_{\Omega_{\varepsilon}} D \mathcal{T}_{\varepsilon}(W) D V d y=\int_{\Omega_{\varepsilon}} W V d y, \quad \forall V \in \mathcal{V}^{\varepsilon},
\end{array}\right.
$$

with $\mathcal{V}_{\varepsilon}$ defined in $(2.2)$, if $\varepsilon>0$.

It is clear that $\mathcal{T}_{\varepsilon}$ is a linear, self-adjoint, positive, and compact operator. Moreover, the eigenvalues of $\mathcal{T}_{0}$ are $\left\{1 / \mu_{k}\right\}_{k \in \mathbb{N}}$ where $\left\{\mu_{k}\right\}_{k \in \mathbb{N}}$ are the eigenvalues of (2.9), while for $\varepsilon>0$ the eigenvalues of $\mathcal{T}_{\varepsilon}$ are $\left\{1 / \lambda_{\varepsilon, k}\right\}_{k \in \mathbb{N}}$ where $\left\{\lambda_{\varepsilon, k}\right\}_{k \in \mathbb{N}}$ are the eigenvalues of (2.1). Consequently, due to Theorem 2.1, $\left\{\mathcal{T}_{\varepsilon}\right\}_{\varepsilon}$ also satisfies (3.1). Then, the statement in Proposition 3.2 follows from Theorem 3.1.

Remark 3.3. Note that this result holds true for a particular sequence. Nevertheless, it is worth mentioning that for any vanishing sequence $\{\varepsilon\} \subset] 0, \min \{1, d\}[$, the existence of a diverging sequence of integer positive numbers $\{k(\varepsilon)\}$ such that

$$
\lim _{\varepsilon \rightarrow 0}\left(\varepsilon^{\gamma} \lambda_{\varepsilon, k(\varepsilon)}\right)=\lambda
$$

can be obtained by means of the convergence of the corresponding spectral families. For the sake of brevity, the proof of this result is not included (see [21], [31], and [32] for the technique).

In order to localize certain sequences of eigenvalues $\lambda_{\varepsilon}=\lambda_{\varepsilon, k(\varepsilon)}$ of $(2.1)$ whose corresponding eigenfunctions do not vanish asymptotically, we first search among those of order $O\left(\varepsilon^{-2}\right)$, namely we look for $\lambda_{\varepsilon}$ eigenvalues of (2.1) such that

$$
\left.\lim _{\varepsilon \rightarrow 0}\left(\varepsilon^{2} \lambda_{\varepsilon}\right)=\lambda \in\right] 0,+\infty[
$$

Moreover, we assume that the corresponding eigenfunctions are normalized by

$$
\left\|U_{\varepsilon}\right\|_{L^{2}\left(\Omega_{\varepsilon}\right)}^{2}=\varepsilon
$$


Thus, rescaling (2.4) leads us to $\left(\lambda_{\varepsilon}, u_{\varepsilon}\right)$, eigenelement of (2.6) with $\lambda_{\varepsilon}$ satisfying (3.2) and $u_{\varepsilon}=\left(u_{\varepsilon}^{a}, u_{\varepsilon}^{b}\right)$ such that

$$
\int_{\Omega^{a}}\left|u_{\varepsilon}^{a}\right|^{2} d x+\int_{\Omega^{b}}\left|u_{\varepsilon}^{b}\right|^{2} d x=1 .
$$

Proposition 3.4 describes the asymptotic behavior, as $\varepsilon \rightarrow 0$, of $\left(\lambda_{\varepsilon}, u_{\varepsilon}\right)$ under the above assumptions, whereas Proposition 3.6 states the behavior of $\left(\lambda_{\varepsilon}, u_{\varepsilon}\right)$ when $\lambda_{\varepsilon}=O\left(\varepsilon^{-\gamma}\right)$ for $\gamma \in] 0,+\infty[\backslash\{2\}$, and (3.4) holds true.

Let $V^{a}$ and $V^{b}$ be the spaces defined by

$$
\begin{aligned}
& V^{a}=\left\{v \in L^{2}\left(\Omega^{a}\right): \partial_{x_{1}} v \in L^{2}\left(\Omega^{a}\right)\right\} \quad \text { and } \\
& V^{b}=\left\{v \in L^{2}\left(\Omega^{b}\right): \partial_{x_{2}} v \in L^{2}\left(\Omega^{b}\right)\right\} .
\end{aligned}
$$

Proposition 3.4. Let $\left(\lambda_{\varepsilon}, u_{\varepsilon}\right)$ be a solution to (2.6) with $\lambda_{\varepsilon}$ satisfying (3.2) and $u_{\varepsilon}=$ $\left(u_{\varepsilon}^{a}, u_{\varepsilon}^{b}\right)$ satisfying (3.4). Let $V^{a}$ and $V^{b}$ be defined in (3.5). Then, there exists a subsequence of $\{\varepsilon\}$, still denoted by $\{\varepsilon\}$, and $\left(u^{a}, u^{b}\right) \in V^{a} \times V^{b}$ (in possible dependence on the subsequence) such that

$$
\left\{\begin{array}{l}
u_{\varepsilon}^{a} \rightarrow u^{a}, \quad \partial_{x_{1}} u_{\varepsilon}^{a} \rightarrow \partial_{x_{1}} u^{a}, \quad \varepsilon \partial_{x_{2}} u_{\varepsilon}^{a} \rightarrow 0 \text { weakly in } L^{2}\left(\Omega^{a}\right), \\
u_{\varepsilon}^{b} \rightarrow u^{b}, \quad \varepsilon \partial_{x_{1}} u_{\varepsilon}^{b} \rightarrow 0, \quad \partial_{x_{2}} u_{\varepsilon}^{b} \rightarrow \partial_{x_{2}} u^{b} \text { weakly in } L^{2}\left(\Omega^{b}\right),
\end{array}\right.
$$

as $\varepsilon$ tends to zero. Moreover, $\left(u^{a}, u^{b}\right)$ satisfies

$$
\left\{\begin{array}{l}
\left(u^{a}, u^{b}\right) \in V^{a} \times V^{b} \\
\int_{\Omega^{a}} \partial_{x_{1}} u^{a} \partial_{x_{1}} v^{a} d x+\int_{\Omega^{b}} \partial_{x_{2}} u^{b} \partial_{x_{2}} v^{b} d x \\
=\lambda\left(\int_{\Omega^{a}} u^{a} v^{a} d x+\int_{\Omega^{b}} u^{b} v^{b} d x\right), \quad \forall\left(v^{a}, v^{b}\right) \in V^{a} \times V^{b},
\end{array}\right.
$$

with $\lambda$ given in (3.2).

Proof. Choosing $\left(v^{a}, v^{b}\right)=\left(u_{\varepsilon}^{a}, u_{\varepsilon}^{b}\right)$ as test function in the equation in (2.6) and taking into account (3.4) give

$$
\int_{\Omega^{a}}\left(\left|\partial_{x_{1}} u_{\varepsilon}^{a}\right|^{2}+\left|\varepsilon \partial_{x_{2}} u_{\varepsilon}^{a}\right|^{2}\right) d x+\int_{\Omega^{b}}\left(\left|\varepsilon \partial_{x_{1}} u_{\varepsilon}^{b}\right|^{2}+\left|\partial_{x_{2}} u_{\varepsilon}^{b}\right|^{2}\right) d x=\varepsilon^{2} \lambda_{\varepsilon}
$$

Then, (3.4), (3.8), and (3.2) provide the existence of a subsequence of $\{\varepsilon\}$, still denoted by $\{\varepsilon\}$, and of $\left(u^{a}, u^{b}\right) \in V^{a} \times V^{b}$ (in possible dependence on the subsequence) satisfying (3.6) (cf. e.g. the argument in (3.15)).

To prove that $\left(u^{a}, u^{b}\right)$ is a solution to problem (3.7), we introduce the spaces

$$
\tilde{V}^{a}=\left\{v^{a} \in C^{\infty}\left(\overline{\Omega^{a}}\right): v^{a}=0 \text { on } \Gamma^{a}\right\} \quad \text { and }
$$




$$
\widetilde{V}^{b}=\left\{v^{b} \in C^{\infty}\left(\overline{\Omega^{b}}\right): v^{b}=0 \text { on } \Gamma^{b}\right\} .
$$

Let $\left(v^{a}, v^{b}\right) \in \widetilde{V}^{a} \times \widetilde{V}^{b}$ be fixed. For each $\varepsilon$, we define

$$
v_{\varepsilon}=\left\{\begin{array}{l}
\left.v^{a}, \text { in } \omega \times\right] \eta_{\varepsilon}, d[ \\
\left.v^{a}\left(x_{1}, \eta_{\varepsilon}\right) \frac{x_{2}}{\eta_{\varepsilon}}+v^{b}\left(\varepsilon x_{1}, 0\right) \frac{\eta_{\varepsilon}-x_{2}}{\eta_{\varepsilon}}, \text { in } \omega \times\right] 0, \eta_{\varepsilon}[ \\
v^{b}, \text { in } \Omega^{b}
\end{array}\right.
$$

where $\left\{\eta_{\varepsilon}\right\}_{\varepsilon}$ is a sequence in $] 0,1[$ such that

$$
\lim _{\varepsilon \rightarrow 0} \frac{\varepsilon}{\sqrt{\eta_{\varepsilon}}}=0
$$

Choosing $\left(v^{a}, v^{b}\right)=\left(\left.v_{\varepsilon}\right|_{\Omega^{a}},\left.v_{\varepsilon}\right|_{\Omega^{b}}\right)$ as test function in (2.6) gives

$$
\begin{aligned}
& \int_{\omega} \int_{\eta_{\varepsilon}}^{d}\left(\partial_{x_{1}} u_{\varepsilon}^{a} \partial_{x_{1}} v^{a}+\varepsilon^{2} \partial_{x_{2}} u_{\varepsilon}^{a} \partial_{x_{2}} v^{a}\right) d x \\
& +\int_{\omega} \int_{0}^{\eta_{\varepsilon}} \partial_{x_{1}} u_{\varepsilon}^{a}\left(\partial_{x_{1}} v^{a}\left(x_{1}, \eta_{\varepsilon}\right) \frac{x_{2}}{\eta_{\varepsilon}}+\varepsilon\left(\partial_{x_{1}} v^{b}\right)\left(\varepsilon x_{1}, 0\right) \frac{\eta_{\varepsilon}-x_{2}}{\eta_{\varepsilon}}\right) d x \\
& +\int_{\omega} \int_{0}^{\eta_{\varepsilon}} \varepsilon^{2} \partial_{x_{2}} u_{\varepsilon}^{a}\left(\frac{v^{a}\left(x_{1}, \eta_{\varepsilon}\right)-v^{b}\left(\varepsilon x_{1}, 0\right)}{\eta_{\varepsilon}}\right) d x \\
& +\int_{\Omega^{b}}\left(\varepsilon^{2} \partial_{x_{1}} u_{\varepsilon}^{b} \partial_{x_{1}} v^{b}+\partial_{x_{2}} u_{\varepsilon}^{b} \partial_{x_{2}} v^{b}\right) d x=\varepsilon^{2} \lambda_{\varepsilon} \int_{\omega} \int_{\eta_{\varepsilon}}^{d} u_{\varepsilon}^{a} v^{a} d x \\
& +\varepsilon^{2} \lambda_{\varepsilon} \int_{\omega} \int_{0}^{\eta_{\varepsilon}} u_{\varepsilon}^{a}\left(v^{a}\left(x_{1}, \eta_{\varepsilon}\right) \frac{x_{2}}{\eta_{\varepsilon}}+v^{b}\left(\varepsilon x_{1}, 0\right) \frac{\eta_{\varepsilon}-x_{2}}{\eta_{\varepsilon}}\right) d x \\
& +\varepsilon^{2} \lambda_{\varepsilon} \int_{\Omega^{b}} u_{\varepsilon}^{b} v^{b} d x .
\end{aligned}
$$

The Cauchy-Schwarz inequality, the fact that $\left(u^{a}, u^{b}\right) \in C^{\infty}\left(\overline{\Omega^{a}}\right) \times C^{\infty}\left(\overline{\Omega^{b}}\right),(3.2),(3.6)$, and (3.10) allow us to pass to the limit, as $\varepsilon$ tends to zero, in (3.11) and obtain

$$
\int_{\Omega^{a}} \partial_{x_{1}} u^{a} \partial_{x_{1}} v^{a} d x+\int_{\Omega^{b}} \partial_{x_{2}} u^{b} \partial_{x_{2}} v^{b} d x=\lambda\left(\int_{\Omega^{a}} u^{a} v^{a} d x+\int_{\Omega^{b}} u^{b} v^{b} d x\right) .
$$

Note that (3.10) is used to prove that the third integral in the left-hand side of (3.11) vanishes as $\varepsilon$ tends to zero.

Finally, equation (3.12) implies that $\left(u^{a}, u^{b}\right)$ satisfies $(3.7)$, since $\left(v^{a}, v^{b}\right)$ in an arbitrary element of $\widetilde{V}^{a} \times \widetilde{V}^{b}$ and this space is dense in $V^{a} \times V^{b}$. 
Corollary 3.5. Let $\lambda$ be a positive real number which is not an eigenvalue of (3.7) and let $\left\{\lambda_{\varepsilon}\right\}_{\varepsilon}$ be any sequence of eigenvalues of (2.6) satisfying (3.2). For each $\varepsilon$, let $u_{\varepsilon}=\left(u_{\varepsilon}^{a}, u_{\varepsilon}^{b}\right)$ be an eigenfunction of (2.6) associated with $\lambda_{\varepsilon}$ and satisfying (3.4). Then

$$
\left\{\begin{array}{l}
u_{\varepsilon}^{a} \rightarrow 0, \quad \partial_{x_{1}} u_{\varepsilon}^{a} \rightarrow 0, \quad \varepsilon \partial_{x_{2}} u_{\varepsilon}^{a} \rightarrow 0 \text { weakly in } L^{2}\left(\Omega^{a}\right) \\
u_{\varepsilon}^{b} \rightarrow 0, \quad \varepsilon \partial_{x_{1}} u_{\varepsilon}^{b} \rightarrow 0, \quad \partial_{x_{2}} u_{\varepsilon}^{b} \rightarrow 0 \text { weakly in } L^{2}\left(\Omega^{b}\right)
\end{array}\right.
$$

as $\varepsilon$ tends to zero.

Proposition 3.6. Assume that $\left\{\lambda_{\varepsilon}\right\}_{\varepsilon}$ is any sequence of eigenvalues of (2.6) such that

$$
\left.\lim _{\varepsilon \rightarrow 0}\left(\varepsilon^{\gamma} \lambda_{\varepsilon}\right)=\lambda \in\right] 0,+\infty[
$$

for some $\gamma \in] 0,+\infty\left[\backslash\{2\}\right.$. For each $\varepsilon$, let $u_{\varepsilon}=\left(u_{\varepsilon}^{a}, u_{\varepsilon}^{b}\right)$ be an eigenfunction of (2.6) associated with $\lambda_{\varepsilon}$ and satisfying (3.4). Then

$$
u_{\varepsilon}^{a} \rightarrow 0 \text { weakly in } L^{2}\left(\Omega^{a}\right), \quad u_{\varepsilon}^{b} \rightarrow 0 \text { weakly in } L^{2}\left(\Omega^{b}\right),
$$

as $\varepsilon$ tends to zero.

Proof. Choosing $\left(v^{a}, v^{b}\right)=\left(\varepsilon^{\gamma-2} u_{\varepsilon}^{a}, \varepsilon^{\gamma-2} u_{\varepsilon}^{b}\right)$ as test function in (2.6) and taking into account (3.4) give

$$
\int_{\Omega^{a}}\left(\varepsilon^{\gamma-2}\left|\partial_{x_{1}} u_{\varepsilon}^{a}\right|^{2}+\varepsilon^{\gamma}\left|\partial_{x_{2}} u_{\varepsilon}^{a}\right|^{2}\right) d x+\int_{\Omega^{b}}\left(\varepsilon^{\gamma}\left|\partial_{x_{1}} u_{\varepsilon}^{b}\right|^{2}+\varepsilon^{\gamma-2}\left|\partial_{x_{2}} u_{\varepsilon}^{b}\right|^{2}\right) d x=\varepsilon^{\gamma} \lambda_{\varepsilon} .
$$

Assume $\gamma \in] 2,+\infty[$. Then, (3.4), (3.14), and (3.13) provide the existence of a subsequence of $\{\varepsilon\}$, still denoted by $\{\varepsilon\}$, and of $\left(u^{a}, u^{b}\right) \in L^{2}\left(\Omega^{a}\right) \times L^{2}\left(\Omega^{b}\right)$ satisfying

$$
\left\{\begin{array}{l}
u_{\varepsilon}^{a} \rightarrow u^{a}, \quad \varepsilon^{(\gamma-2) / 2} \partial_{x_{1}} u_{\varepsilon}^{a} \rightarrow 0, \quad \varepsilon^{\gamma / 2} \partial_{x_{2}} u_{\varepsilon}^{a} \rightarrow 0 \text { weakly in } L^{2}\left(\Omega^{a}\right), \\
u_{\varepsilon}^{b} \rightarrow u^{b}, \quad \varepsilon^{\gamma / 2} \partial_{x_{1}} u_{\varepsilon}^{b} \rightarrow 0, \quad \varepsilon^{(\gamma-2) / 2} \partial_{x_{2}} u_{\varepsilon}^{b} \rightarrow 0 \text { weakly in } L^{2}\left(\Omega^{b}\right)
\end{array}\right.
$$

as $\varepsilon$ tends to zero. Note that the convergence of the scaled derivatives towards zero follows from the convergence of $\left(u_{\varepsilon}^{a}, u_{\varepsilon}^{b}\right)$ towards $\left(u^{a}, u^{b}\right)$ in $\mathcal{D}^{\prime}\left(\Omega^{a}\right) \times \mathcal{D}^{\prime}\left(\Omega^{b}\right)$. Consequently, passing to the limit in (2.6), as $\varepsilon$ tends to zero, with test functions $\left(\varepsilon^{\gamma-2} v^{a}, \varepsilon^{\gamma-2} v^{b}\right)$, $\left(v^{a}, v^{b}\right) \in C_{0}^{\infty}\left(\Omega^{a}\right) \times C_{0}^{\infty}\left(\Omega^{b}\right)$, and recalling $\lambda \neq 0$ provide

$$
u^{a}=0 \text { in } \Omega^{a}, \quad u^{b}=0 \text { in } \Omega^{b},
$$

which concludes the proof when $\gamma \in] 2,+\infty[$.

Assume $\gamma \in] 0,2\left[\right.$. Choosing $\left(v^{a}, v^{b}\right)=\left(u_{\varepsilon}^{a}, u_{\varepsilon}^{b}\right)$ as test function in (2.6) and taking into account (3.13) give

$$
\int_{\Omega^{a}}\left(\left|\partial_{x_{1}} u_{\varepsilon}^{a}\right|^{2}+\left|\varepsilon \partial_{x_{2}} u_{\varepsilon}^{a}\right|^{2}\right) d x+\int_{\Omega^{b}}\left(\left|\varepsilon \partial_{x_{1}} u_{\varepsilon}^{b}\right|^{2}+\left|\partial_{x_{2}} u_{\varepsilon}^{b}\right|^{2}\right) d x=\varepsilon^{2-\gamma} \varepsilon^{\gamma} \lambda_{\varepsilon} \rightarrow 0
$$


as $\varepsilon$ tends to zero. Consequently, taking again into account (3.4), there exists a subsequence of $\{\varepsilon\}$, still denoted by $\{\varepsilon\}$, and $u^{a} \in L^{2}\left(\Omega^{a}\right)$, independent of $x_{1}, u^{b} \in L^{2}\left(\Omega^{b}\right)$, independent of $x_{2}$, such that

$$
\left\{\begin{array}{l}
u_{\varepsilon}^{a} \rightarrow u^{a} \text { weakly in } L^{2}\left(\Omega^{a}\right), \quad \partial_{x_{1}} u_{\varepsilon}^{a} \rightarrow 0 \text { strongly in } L^{2}\left(\Omega^{a}\right), \\
u_{\varepsilon}^{b} \rightarrow u^{b} \text { weakly in } L^{2}\left(\Omega^{b}\right), \quad \partial_{x_{2}} u_{\varepsilon}^{b} \rightarrow 0 \text { strongly in } L^{2}\left(\Omega^{b}\right),
\end{array}\right.
$$

as $\varepsilon$ tends to zero. Furthermore, from (3.14) we deduce

$$
\varepsilon^{\gamma / 2} \partial_{x_{2}} u_{\varepsilon}^{a} \rightarrow 0 \text { weakly in } L^{2}\left(\Omega^{a}\right), \quad \varepsilon^{\gamma / 2} \partial_{x_{1}} u_{\varepsilon}^{b} \rightarrow 0 \text { weakly in } L^{2}\left(\Omega^{b}\right) .
$$

Finally, passing to the limit in (2.6), as $\varepsilon$ tends to zero, with test functions $\left(\varepsilon^{\gamma-2} v^{a}, \varepsilon^{\gamma-2} v^{b}\right)$, $\left(v^{a}, v^{b}\right) \in C_{0}^{\infty}(] 0, d[) \times C_{0}^{\infty}(\omega \backslash\{0\})$, and recalling that $\lambda \neq 0$ provide

$$
u^{a}=0 \text { in } \Omega^{a}, \quad u^{b}=0 \text { in } \Omega^{b},
$$

which concludes the proof when $\gamma \in] 0,2[$.

\section{Analysis of the spectral limit problem (3.7) and be- yond}

In this section we present an analysis of the eigenvalues and eigenfunctions of limit problem (3.7) obtained in Section 3. We give a characterization of the eigenvalues and eigenfunctions assuming some hypotheses of convergence in addition to those in Proposition 3.4 (see also (3.2)). These hypotheses are justified in Section 5. In particular, Propositions 4.1 and 4.2 below relate the eigenvalues and eigenfunctions of problem (3.7) with those of the $1 D$ Neumann problems (1.3) and (1.4) while, introducing some kind of corrector term for the eigenvalues, in Subsection 4.1 we establish an additional relation of the eigenvalues and eigenfunctions of (3.7) with those of the $1 D$ Dirichlet problems (1.6) and (1.7).

It is evident that the spectral problem (3.7) can be split in the following two uncoupled problems

$$
\begin{aligned}
& \left\{\begin{array}{l}
u^{a} \in V^{a}, \\
\int_{\Omega^{a}} \partial_{x_{1}} u^{a} \partial_{x_{1}} v d x=\lambda^{a} \int_{\Omega^{a}} u^{a} v d x, \quad \forall v \in V^{a},
\end{array}\right. \\
& \left\{\begin{array}{l}
u^{b} \in V^{b}, \\
\int_{\Omega^{b}} \partial_{x_{2}} u^{b} \partial_{x_{2}} v d x=\lambda^{b} \int_{\Omega^{b}} u^{b} v d x, \quad \forall v \in V^{b},
\end{array}\right.
\end{aligned}
$$

i.e. if $A, B$, and $C$ denote the sets of the eigenvalues of (4.1), (4.2), and (3.7), respectively, then

$$
C=A \cup B
$$


Proposition 4.1. The couple $\left(\lambda^{a}, u^{a}\right)$ is an eigenelement of (4.1) iff $\lambda^{a}$ is an eigenvalue of (1.3) and

$$
u^{a}\left(x_{1}, x_{2}\right)=\alpha\left(x_{2}\right) z^{a}\left(x_{1}\right) \text { in } \Omega^{a},
$$

with $\alpha \in L^{2}(] 0, d[)$ and $z^{a}$ eigenfunction of (1.3) associated with $\lambda^{a}$.

Proof. Choosing $v=\varphi \psi$, with $\psi \in C_{0}^{\infty}(] 0, d[)$ and $\varphi \in H^{1}(\omega)$, as test function in (4.1) gives

$$
\int_{0}^{d}\left(\int_{\omega}\left(\partial_{x_{1}} u^{a} \partial_{x_{1}} \varphi-\lambda^{a} u^{a} \varphi\right) d x_{1}\right) \psi d x_{2}=0, \quad \forall \varphi \in H^{1}(\omega), \forall \psi \in C_{0}^{\infty}(] 0, d[),
$$

which implies

$$
\int_{\omega} \partial_{x_{1}} u^{a}\left(x_{1}, x_{2}\right) \partial_{x_{1}} \varphi\left(x_{1}\right) d x_{1}=\lambda^{a} \int_{\omega} u^{a}\left(x_{1}, x_{2}\right) \varphi\left(x_{1}\right) d x_{1}, \forall \varphi \in H^{1}(\omega),
$$

for a.e. $\left.x_{2} \in\right] 0, d[$, i.e.

$$
\left\{\begin{array}{l}
-\partial_{x_{1}}^{2} u^{a}\left(x_{1}, x_{2}\right)=\lambda^{a} u^{a}\left(x_{1}, x_{2}\right), \text { in } \omega, \\
\partial_{x_{1}} u^{a}\left(\cdot, x_{2}\right)=0, \text { on } \partial \omega,
\end{array} \quad \text { for a.e. } x_{2} \in\right] 0, d[.
$$

Finally, the result follows from (4.3) by the classical decomposition method.

Similarly, the following result holds true.

Proposition 4.2. The couple $\left(\lambda^{b}, u^{b}\right)$ is an eigenelement of problem (4.2) iff $\lambda^{b}$ is an eigenvalue of (1.4) and

$$
u^{b}\left(x_{1}, x_{2}\right)=\beta\left(x_{1}\right) z^{b}\left(x_{2}\right) \text { in } \Omega^{b}
$$

with $\beta \in L^{2}(\omega)$ and $z^{b}$ eigenfunction of (1.4) associated with $\lambda^{b}$.

As it is well known, all the eigenvalues of Neumann problem (1.3) are simple and satisfy

$$
0=\lambda_{0}^{a}<\lambda_{1}^{a}<\cdots<\lambda_{k}^{a}<\cdots \stackrel{k \rightarrow+\infty}{\longrightarrow}+\infty,
$$

Moreover, they can be explicitly computed in terms of the extremes of the interval $\omega$. We denote by $\left\{z_{k}^{a}\right\}_{k \in \mathbb{N}_{0}}, \mathbb{N}_{0}=\{0\} \cup \mathbb{N}$, the corresponding eigenfunctions subject to the orthonormalization condition

$$
\int_{\omega} z_{i}^{a} z_{j}^{a} d x_{1}=\delta_{i j} \quad i, j \in \mathbb{N}_{0}
$$

Note that $z_{0}^{a}=|\omega|^{-\frac{1}{2}}$, since $\lambda_{0}^{a}=0$.

Similarly, the set of all the eigenvalues of the Neumann problem (1.4) is given by

$$
\left\{(k \pi)^{2}\right\}_{k \in \mathbb{N}_{0}}
$$

while

$$
\{1\} \cup\left\{\sqrt{2} \cos \left(k \pi x_{2}\right)\right\}_{k \in \mathbb{N}}
$$

is an orthonormal basis in $L^{2}(]-1,0[), z_{k}^{b}=\sqrt{2} \cos \left(k \pi x_{2}\right)$ being an eigenfunction of (1.4) associated with the eigenvalue $\lambda_{k}^{b}=(k \pi)^{2}$, for $k \in \mathbb{N}$, and 1 associated with 0 . 
Corollary 4.3. Let $\lambda_{\varepsilon}$ be an eigenvalue of problem (2.6) with $\left\{\lambda_{\varepsilon}\right\}_{\varepsilon} \subset \mathbb{R}$ satisfying (3.2). Then, $\lambda$ defined by (3.2) is an eigenvalue of problem (3.7) iff

$$
\lambda \in\left\{\lambda_{k}^{a}\right\}_{k \in \mathbb{N}} \cup\left\{\lambda_{k}^{b}\right\}_{k \in \mathbb{N}} .
$$

Proof. This corollary is an immediate consequence of previous results.

Remark 4.4. If

then in Proposition 3.4

$$
\lambda \notin\left\{\lambda_{k}^{a}\right\}_{k \in \mathbb{N}_{0}} \cup\left\{\lambda_{k}^{b}\right\}_{k \in \mathbb{N}_{0}}
$$

$$
u^{a}=0 \text { in } \Omega^{a}, \quad u^{b}=0 \text { in } \Omega^{b},
$$

and (3.6) holds for the whole sequence.

According to Proposition 4.1 and Proposition 4.2, if $\lambda \in\left\{\lambda_{k}^{a}\right\}_{k \in \mathbb{N}} \cup\left\{\lambda_{k}^{b}\right\}_{k \in \mathbb{N}}$, the limit eigenfunction $\left(u^{a}, u^{b}\right)$ in the statement of Proposition 3.4 can be written as

$$
u^{a}\left(x_{1}, x_{2}\right)=\alpha\left(x_{2}\right) z^{a}\left(x_{1}\right) \text { in } \Omega^{a}, \quad u^{b}\left(x_{1}, x_{2}\right)=\beta\left(x_{1}\right) z^{b}\left(x_{2}\right) \text { in } \Omega^{b},
$$

where $z^{a}$ is an eigenfunction of (1.3) associated with $\lambda^{a}, z^{b}$ is an eigenfunction of (1.4) associated with $\lambda^{b}$, and $\alpha \in L^{2}(] 0, d[)$ and $\beta \in L^{2}(\omega)$ are unknown functions (here, we assume that $\alpha$ or $\beta$ are the zero function if $\lambda \notin\left\{\lambda_{k}^{a}\right\}_{k \in \mathbb{N}}$ or $\lambda \notin\left\{\lambda_{k}^{b}\right\}_{k \in \mathbb{N}}$, respectively). There are many possible functions $\alpha\left(x_{2}\right)$ or $\beta\left(x_{1}\right)$ such that $u^{a}$ and $u^{b}$ given in (4.8) satisfy (3.7). In the next subsection, we look for particular functions $\alpha\left(x_{2}\right)$ and $\beta\left(x_{1}\right)$ that will be used in Section 5 for proving the main results.

\subsection{The correction terms}

Proposition 4.5. For each $\varepsilon$, let $\left(\lambda_{\varepsilon}, u_{\varepsilon}\right)$ be a solution to problem (2.6) with $\lambda_{\varepsilon}$ satisfying the following property:

$$
\exists \lambda \in\left\{\lambda_{k}^{a}\right\}_{k \in \mathbb{N}} \cup\left\{\lambda_{k}^{b}\right\}_{k \in \mathbb{N}}, \quad \exists \mu \in \mathbb{R} \quad: \quad \lim _{\varepsilon \rightarrow 0} \frac{\varepsilon^{2} \lambda_{\varepsilon}-\lambda}{\varepsilon^{2}}=\mu,
$$

where $\left\{\lambda_{k}^{a}\right\}_{k \in \mathbb{N}}$ is the sequence of all the eigenvalues of (1.3) (see (4.4)) and $\left\{\lambda_{k}^{b}\right\}_{k \in \mathbb{N}}$ is the sequence of all the eigenvalues of (1.4) (see (4.6)). Moreover, assume that $u_{\varepsilon}=\left(u_{\varepsilon}^{a}, u_{\varepsilon}^{b}\right)$ satisfies (3.4). Consider a subsequence of $\{\varepsilon\}$, still denoted by $\{\varepsilon\}$, and $\left(u^{a}, u^{b}\right) \in V^{a} \times V^{b}$ satisfying (3.6) and (3.7). In addition, assume that

$$
\left\{\begin{array}{l}
\left\{\frac{u_{\varepsilon}^{a}-u^{a}}{\varepsilon}\right\}_{\varepsilon} \text { is bounded in } L^{2}\left(\Omega^{a}\right), \\
\left\{\frac{u_{\varepsilon}^{b}-u^{b}}{\varepsilon}\right\}_{\varepsilon} \text { is bounded in } L^{2}\left(\Omega^{b}\right) .
\end{array}\right.
$$

Then,

$$
\left\{\begin{array}{l}
\exists m \in \mathbb{N}: u^{a}=\alpha\left(x_{2}\right) z_{m}^{a}\left(x_{1}\right), \text { in } \Omega^{a}, \\
\exists n \in \mathbb{N}: u^{b}=\beta\left(x_{1}\right) z_{n}^{b}\left(x_{2}\right), \text { in } \Omega^{b},
\end{array}\right.
$$


with $z_{k}^{a}$ an eigenfunction of (1.3) associated with $\lambda_{k}^{a}$ and satisfying (4.5), $\left\{z_{k}^{b}\right\}_{k \in \mathbb{N}}$ given in (4.7), $\alpha$ and $\beta$ satisfying (1.6) and (1.7), respectively, where $\mu$ is given in (4.9), and $\alpha$ or $\beta$ different from zero.

Proof. The proof of this proposition will be developed in several steps.

The first step is devoted to improving Proposition 3.4, i.e. to proving that

$$
\left\{\begin{array}{l}
u_{\varepsilon}^{a} \rightarrow u^{a} \text { weakly in } H^{1}\left(\Omega^{a}\right), \\
u_{\varepsilon}^{b} \rightarrow u^{b} \text { weakly in } H^{1}\left(\Omega^{b}\right),
\end{array}\right.
$$

as $\varepsilon$ tends to zero,

$$
\begin{aligned}
& \left\{\begin{array}{l}
\left\{\frac{1}{\varepsilon} \partial_{x_{1}}\left(u_{\varepsilon}^{a}-u^{a}\right)\right\}_{\varepsilon} \text { is bounded in } L^{2}\left(\Omega^{a}\right), \\
\left\{\frac{1}{\varepsilon} \partial_{x_{2}}\left(u_{\varepsilon}^{b}-u^{b}\right)\right\}_{\varepsilon} \text { is bounded in } L^{2}\left(\Omega^{b}\right),
\end{array}\right. \\
& \int_{\Omega^{a}}\left|u^{a}\right|^{2} d x+\int_{\Omega^{b}}\left|u^{b}\right|^{2} d x=1,
\end{aligned}
$$

and

$$
u^{a}=0, \text { on } \Gamma^{a}, \quad u^{b}=0, \text { on } \Gamma^{b} .
$$

To this aim, choosing $\left(v^{a}, v^{b}\right)=\left(\frac{u_{\varepsilon}^{a}}{\varepsilon^{2}}, \frac{u_{\varepsilon}^{b}}{\varepsilon^{2}}\right)$ as test function in the equation in (2.6) and taking into account (3.4) give

$$
\int_{\Omega^{a}}\left(\left|\frac{1}{\varepsilon} \partial_{x_{1}} u_{\varepsilon}^{a}\right|^{2}+\left|\partial_{x_{2}} u_{\varepsilon}^{a}\right|^{2}\right) d x+\int_{\Omega^{b}}\left(\left|\partial_{x_{1}} u_{\varepsilon}^{b}\right|^{2}+\left|\frac{1}{\varepsilon} \partial_{x_{2}} u_{\varepsilon}^{b}\right|^{2}\right) d x=\frac{\varepsilon^{2} \lambda_{\varepsilon}}{\varepsilon^{2}} .
$$

Moreover, choosing $\left(v^{a}, v^{b}\right)=\left(-\frac{u_{\varepsilon}^{a}}{\varepsilon^{2}},-\frac{u_{\varepsilon}^{b}}{\varepsilon^{2}}\right)$ as test function in the equation in (3.7) gives

$$
\begin{aligned}
& -\int_{\Omega^{a}} \frac{1}{\varepsilon^{2}} \partial_{x_{1}} u^{a} \partial_{x_{1}} u_{\varepsilon}^{a} d x-\int_{\Omega^{b}} \frac{1}{\varepsilon^{2}} \partial_{x_{2}} u^{b} \partial_{x_{2}} u_{\varepsilon}^{b} d x \\
& =-\frac{\lambda}{\varepsilon^{2}}\left(\int_{\Omega^{a}} u^{a} u_{\varepsilon}^{a} d x+\int_{\Omega^{b}} u^{b} u_{\varepsilon}^{b} d x\right) .
\end{aligned}
$$

Then, adding (4.16) to (4.17) and taking again into account (3.4) provide

$$
\begin{aligned}
& \int_{\Omega^{a}}\left(\frac{1}{\varepsilon^{2}} \partial_{x_{1}} u_{\varepsilon}^{a} \partial_{x_{1}}\left(u_{\varepsilon}^{a}-u^{a}\right)+\left|\partial_{x_{2}} u_{\varepsilon}^{a}\right|^{2}\right) d x \\
& +\int_{\Omega^{b}}\left(\left|\partial_{x_{1}} u_{\varepsilon}^{b}\right|^{2}+\frac{1}{\varepsilon^{2}} \partial_{x_{2}} u_{\varepsilon}^{b} \partial_{x_{2}}\left(u_{\varepsilon}^{b}-u^{b}\right)\right) d x \\
& =\frac{\varepsilon^{2} \lambda_{\varepsilon}-\lambda}{\varepsilon^{2}}+\frac{\lambda}{\varepsilon^{2}}\left(\int_{\Omega^{a}} u_{\varepsilon}^{a}\left(u_{\varepsilon}^{a}-u^{a}\right) d x+\int_{\Omega^{b}} u_{\varepsilon}^{b}\left(u_{\varepsilon}^{b}-u^{b}\right) d x\right) .
\end{aligned}
$$


Now, choosing $\left(v^{a}, v^{b}\right)=\left(-\frac{u_{\varepsilon}^{a}-u^{a}}{\varepsilon^{2}},-\frac{u_{\varepsilon}^{b}-u^{b}}{\varepsilon^{2}}\right)$ as test function in the equation in (3.7) gives

$$
\begin{aligned}
& -\int_{\Omega^{a}} \frac{1}{\varepsilon^{2}} \partial_{x_{1}} u^{a} \partial_{x_{1}}\left(u_{\varepsilon}^{a}-u^{a}\right) d x-\int_{\Omega^{b}} \frac{1}{\varepsilon^{2}} \partial_{x_{2}} u^{b} \partial_{x_{2}}\left(u_{\varepsilon}^{b}-u^{b}\right) d x \\
& =-\frac{\lambda}{\varepsilon^{2}}\left(\int_{\Omega^{a}} u^{a}\left(u_{\varepsilon}^{a}-u^{a}\right) d x+\int_{\Omega^{b}} u^{b}\left(u_{\varepsilon}^{b}-u^{b}\right) d x\right) .
\end{aligned}
$$

Finally, adding (4.18) to (4.19) provides

$$
\begin{aligned}
& \int_{\Omega^{a}}\left(\left|\frac{1}{\varepsilon} \partial_{x_{1}}\left(u_{\varepsilon}^{a}-u^{a}\right)\right|^{2}+\left|\partial_{x_{2}} u_{\varepsilon}^{a}\right|^{2}\right) d x+\int_{\Omega^{b}}\left(\left|\partial_{x_{1}} u_{\varepsilon}^{b}\right|^{2}+\left|\frac{1}{\varepsilon} \partial_{x_{2}}\left(u_{\varepsilon}^{b}-u^{b}\right)\right|^{2}\right) d x \\
& =\frac{\varepsilon^{2} \lambda_{\varepsilon}-\lambda}{\varepsilon^{2}}+\lambda\left(\int_{\Omega^{a}}\left|\frac{u_{\varepsilon}^{a}-u^{a}}{\varepsilon}\right|^{2} d x+\int_{\Omega^{b}}\left|\frac{u_{\varepsilon}^{b}-u^{b}}{\varepsilon}\right|^{2} d x\right),
\end{aligned}
$$

which combined with assumptions (4.9) and (4.10) proves (4.12)-(4.15).

The second step is devoted to proving the decompositions in (4.11) with $\alpha$ and $\beta$ satisfying the equations in (1.6) and (1.7), respectively, where $\mu$ is given in (4.9).

As far as the first decomposition in (4.11) is concerned, if $\lambda$ is not an eigenvalue of problem (4.1), then

$$
u^{a}=0 \text { in } \Omega^{a} .
$$

Consequently, the first decomposition in (4.11) holds true for any $m \in \mathbb{N}$ with $\alpha=0$ (also for $m=0)$.

If $\left(\lambda, u^{a}\right)$ is an eigenelement of problem (4.1), Proposition 4.1 (cf. also (4.4) and (4.5)) ensures that

$$
\exists m \in \mathbb{N}: \lambda=\lambda_{m}^{a}, \quad u^{a}=\alpha\left(x_{2}\right) z_{m}^{a}\left(x_{1}\right), \text { in } \Omega^{a},
$$

with some function $\alpha \in L^{2}(] 0, d[)$. More precisely, due to (4.12), $\alpha \in H^{1}(] 0, d[)$. Then, the aim is to prove that

$$
\int_{0}^{d} \alpha^{\prime} \psi^{\prime} d x_{2}=\mu \int_{0}^{d} \alpha \psi d x_{2}, \quad \forall \psi \in C_{0}^{\infty}(] 0, d[),
$$

where $\mu$ is given in (4.9).

Subtracting equation in (3.7) by equation in (2.6) gives

$$
\left\{\begin{array}{l}
\int_{\Omega^{a}}\left(\partial_{x_{1}}\left(u_{\varepsilon}^{a}-u^{a}\right) \partial_{x_{1}} v^{a}+\varepsilon^{2} \partial_{x_{2}} u_{\varepsilon}^{a} \partial_{x_{2}} v^{a}\right) d x \\
+\int_{\Omega^{b}}\left(\varepsilon^{2} \partial_{x_{1}} u_{\varepsilon}^{b} \partial_{x_{1}} v^{b}+\partial_{x_{2}}\left(u_{\varepsilon}^{b}-u^{b}\right) \partial_{x_{2}} v^{b}\right) d x \\
=\left(\varepsilon^{2} \lambda_{\varepsilon}-\lambda\right)\left(\int_{\Omega^{a}} u_{\varepsilon}^{a} v^{a} d x+\int_{\Omega^{b}} u_{\varepsilon}^{b} v^{b} d x\right) \\
+\lambda\left(\int_{\Omega^{a}}\left(u_{\varepsilon}^{a}-u^{a}\right) v^{a} d x+\int_{\Omega^{b}}\left(u_{\varepsilon}^{b}-u^{b}\right) v^{b} d x\right), \quad \forall\left(v^{a}, v^{b}\right) \in V_{\varepsilon} .
\end{array}\right.
$$


Now, choosing $\left(v^{a}, v^{b}\right)=\left(\frac{z_{m}^{a}\left(x_{1}\right) \psi\left(x_{2}\right)}{\varepsilon^{2}}, 0\right)$ as test function in $(4.22)$, with $\psi \in C_{0}^{\infty}(] 0, d[)$, gives

$$
\left\{\begin{array}{l}
\int_{\Omega^{a}}\left(\frac{1}{\varepsilon^{2}} \partial_{x_{1}}\left(\psi\left(x_{2}\right) u_{\varepsilon}^{a}-\psi\left(x_{2}\right) u^{a}\right)\left(z_{m}^{a}\right)^{\prime}\left(x_{1}\right)+\partial_{x_{2}} u_{\varepsilon}^{a} z_{m}^{a}\left(x_{1}\right) \psi^{\prime}\left(x_{2}\right)\right) d x \\
=\frac{\varepsilon^{2} \lambda_{\varepsilon}-\lambda}{\varepsilon^{2}} \int_{\Omega^{a}} u_{\varepsilon}^{a} z_{m}^{a}\left(x_{1}\right) \psi\left(x_{2}\right) d x \\
+\lambda \int_{\Omega^{a}} \frac{1}{\varepsilon^{2}}\left(u_{\varepsilon}^{a}-u^{a}\right) z_{m}^{a}\left(x_{1}\right) \psi\left(x_{2}\right) d x, \quad \forall \psi \in C_{0}^{\infty}(] 0, d[) .
\end{array}\right.
$$

On the other side, since $z_{m}^{a}$ satisfies problem (1.3), one has

$$
\left\{\begin{array}{l}
\int_{\Omega^{a}} \frac{1}{\varepsilon^{2}} \partial_{x_{1}}\left(\psi\left(x_{2}\right) u_{\varepsilon}^{a}-\psi\left(x_{2}\right) u^{a}\right)\left(z_{m}^{a}\right)^{\prime}\left(x_{1}\right) d x \\
=\lambda \int_{\Omega^{a}} \frac{1}{\varepsilon^{2}}\left(u_{\varepsilon}^{a}-u^{a}\right) z_{m}^{a}\left(x_{1}\right) \psi\left(x_{2}\right) d x, \quad \forall \psi \in C_{0}^{\infty}(] 0, d[) .
\end{array}\right.
$$

Consequently, from (4.23) and (4.24) one derives

$$
\int_{\Omega^{a}} \partial_{x_{2}} u_{\varepsilon}^{a} z_{m}^{a}\left(x_{1}\right) \psi^{\prime}\left(x_{2}\right) d x=\frac{\varepsilon^{2} \lambda_{\varepsilon}-\lambda}{\varepsilon^{2}} \int_{\Omega^{a}} u_{\varepsilon}^{a} z_{m}^{a}\left(x_{1}\right) \psi\left(x_{2}\right) d x, \forall \psi \in C_{0}^{\infty}(] 0, d[) .
$$

Finally, passing to the limit in (4.25), as $\varepsilon$ tends to zero, and using (4.9) and (4.12) imply

$$
\int_{\Omega^{a}} \partial_{x_{2}} u^{a} z_{m}^{a}\left(x_{1}\right) \psi^{\prime}\left(x_{2}\right) d x=\mu \int_{\Omega^{a}} u^{a} z_{m}^{a}\left(x_{1}\right) \psi\left(x_{2}\right) d x, \quad \forall \psi \in C_{0}^{\infty}(] 0, d[),
$$

which proves (4.21), since

$$
u^{a}=z_{m}^{a}\left(x_{1}\right) \alpha\left(x_{2}\right) \text { in } \Omega^{a}, \quad \text { and } \quad \int_{\omega}\left|z_{m}^{a}\left(x_{1}\right)\right|^{2} d x_{1}=1 .
$$

As far as the second decomposition in (4.11) is concerned, if $\lambda$ is not an eigenvalue of problem (4.2), then

$$
u^{b}=0 \text { in } \Omega^{b} .
$$

Consequently, the second decomposition in (4.11) holds true for any $n \in \mathbb{N}$ with $\beta=0$.

If $\left(\lambda, u^{b}\right)$ is an eigenelement of problem (4.2), Proposition 4.2 (cf. also (4.6) and (4.7)) ensures that

$$
\exists n \in \mathbb{N}: \lambda=\lambda_{n}^{b}, \quad u^{b}=\beta\left(x_{1}\right) z_{n}^{b}\left(x_{2}\right), \text { in } \Omega^{b},
$$

with some function $\beta \in L^{2}(\omega)$. More precisely, due to (4.12), $\beta \in H^{1}(\omega)$. For $\varepsilon$ small enough, choosing $\left(v^{a}, v^{b}\right)=\left(0, \frac{\chi\left(x_{1}\right) z_{n}^{b}\left(x_{2}\right)}{\varepsilon^{2}}\right)$ as test function in (4.22), with $\chi \in C_{0}^{\infty}(\omega \backslash\{0\})$ and arguing as above provide

$$
\int_{\omega} \beta^{\prime} \chi^{\prime} d x_{1}=\mu \int_{\omega} \beta \chi d x_{1}, \quad \forall \chi \in C_{0}^{\infty}(\omega \backslash\{0\}),
$$


where $\mu$ is given in (4.9).

The third step is devoted to proving the boundary conditions in (1.6) and (1.7), i.e.

$$
\begin{gathered}
\alpha(d)=0, \quad \beta=0, \text { on } \partial \omega, \\
\beta(0)=0 \\
\alpha(0)=0 .
\end{gathered}
$$

Identifications (4.28) follow from (4.15), (4.20), (4.27), and the fact that $z_{m}^{a}$ and $z_{n}^{b}$ are not the zero function.

To identify the other boundary conditions, we first show that

$$
\beta(0) z_{n}^{b}(0) \int_{\omega} g\left(x_{1}\right) d x_{1}=\alpha(0) \int_{\omega} z_{m}^{a}\left(x_{1}\right) g\left(x_{1}\right) d x_{1}, \quad \forall g \in C^{1}(\bar{\omega}) .
$$

On one hand, the second lines in (4.12) and (4.13) allow us to apply Proposition 2.1 in [12] to the sequence $\left\{\left|u_{\varepsilon}^{b}-u^{b}\right|\right\}_{\varepsilon}$ and to obtain

$$
\lim _{\varepsilon \rightarrow 0} \int_{\omega}\left|u_{\varepsilon}^{b}\left(\varepsilon x_{1}, 0\right)-u^{b}\left(\varepsilon x_{1}, 0\right)\right| d x_{1}=0
$$

which implies

$$
\lim _{\varepsilon \rightarrow 0} \int_{\omega}\left(u_{\varepsilon}^{b}\left(\varepsilon x_{1}, 0\right)-u^{b}\left(\varepsilon x_{1}, 0\right)\right) g\left(x_{1}\right) d x_{1}=0, \quad \forall g \in C^{1}(\bar{\omega}) .
$$

Moreover, (4.27) and the continuity of $\beta$ (since $\beta \in H^{1}(\omega)$ ), ensure that

$$
\begin{aligned}
& \limsup _{\varepsilon \rightarrow 0} \int_{\omega}\left|\left(u^{b}\left(\varepsilon x_{1}, 0\right)-\beta(0) z_{n}^{b}(0)\right) g\left(x_{1}\right)\right| d x_{1} \\
& \leq\left|z_{n}^{b}(0)\right| \max _{\bar{\omega}}|g| \lim _{\varepsilon \rightarrow 0} \int_{\omega}\left|\beta\left(\varepsilon x_{1}\right)-\beta(0)\right| d x_{1}=0, \quad \forall g \in C^{1}(\bar{\omega}) .
\end{aligned}
$$

Combining (4.32) and (4.33) proves

$$
\lim _{\varepsilon \rightarrow 0} \int_{\omega} u_{\varepsilon}^{b}\left(\varepsilon x_{1}, 0\right) g\left(x_{1}\right) d x_{1}=\beta(0) z_{n}^{b}(0) \int_{\omega} g\left(x_{1}\right) d x_{1}, \quad \forall g \in C^{1}(\bar{\omega}) .
$$

On the other hand, the first line in (4.12) and (4.20) provide

$$
\begin{aligned}
& \lim _{\varepsilon \rightarrow 0} \int_{\omega} u_{\varepsilon}^{a}\left(x_{1}, 0\right) g\left(x_{1}\right) d x_{1}=\int_{\omega} u^{a}\left(x_{1}, 0\right) g\left(x_{1}\right) d x_{1} \\
& =\alpha(0) \int_{\omega} z_{m}^{a}\left(x_{1}\right) g\left(x_{1}\right) d x_{1}, \quad \forall g \in C^{1}(\bar{\omega}) .
\end{aligned}
$$

Finally, (4.31) follows from (4.34), (4.35), and the fact that

$$
u_{\varepsilon}^{a}\left(x_{1}, 0\right)=u_{\varepsilon}^{b}\left(\varepsilon x_{1}, 0\right), \text { on } \omega .
$$


The proof of (4.29) depends on $\lambda$; more precisely, if it is an eigenvalue of (4.1) or not. At first note that (see (4.7))

$$
z_{n}^{b}(0) \neq 0, \quad \forall n \in \mathbb{N}_{0} .
$$

If $\lambda$ is not an eigenvalue of (4.1), then

$$
u^{a}=0 \text { in } \Omega^{a} .
$$

Thus, as above remarked, $\alpha$ is the zero function. Consequently, choosing

$$
g\left(x_{1}\right)=1, \text { in } \bar{\omega}
$$

in (4.31) gives

$$
\beta(0)=0 .
$$

If $\lambda$ is an eigenvalue of (4.1), choosing

$$
g\left(x_{1}\right)=z_{0}^{a}\left(x_{1}\right)=|\omega|^{-\frac{1}{2}}, \text { in } \bar{\omega},
$$

in (4.31) gives

$$
\beta(0) z_{n}^{b}(0)|\omega|^{\frac{1}{2}}=\alpha(0) \int_{\omega} z_{m}^{a}\left(x_{1}\right) z_{0}^{a}\left(x_{1}\right) d x_{1}=0,
$$

since $\left\{z_{k}^{a}\right\}_{k \in \mathbb{N}_{0}}$ is an orthonormal basis in $L^{2}(\omega)$ and $m>0$ (we explicitly remark that $m>0$ because $\lambda \neq 0$ ). Then, (4.29) follows from (4.37) and (4.38).

Finally, (4.30) is obtained choosing

$$
g\left(x_{1}\right)=z_{m}^{a}\left(x_{1}\right), \text { in } \bar{\omega},
$$

in (4.31), using (4.29) and recalling that $\left\{z_{k}^{a}\right\}_{k \in \mathbb{N}_{0}}$ is an orthonormal basis in $L^{2}(\omega)$.

Remark 4.6. Notice that $k \neq 0$ is assumed in (4.9). This assumption is equivalent to requiring $\lambda \neq 0$. Nevertheless, we note that the proof of Proposition 4.5 up to (4.31) also works if $k=0$ in (4.9), i.e. if $\lambda=0$. We emphasize that the case $\lambda=0$ will be treated in Proposition 4.7, without assumption (4.10).

Proposition 4.7. For each $\varepsilon$, let $\left(\lambda_{\varepsilon}, u_{\varepsilon}\right)$ be a solution to (2.6) with $\lambda_{\varepsilon}$ satisfying the following property:

$$
\exists \mu \in] 0,+\infty\left[\quad: \quad \lim _{\varepsilon \rightarrow 0} \lambda_{\varepsilon}=\mu,\right.
$$

and $u_{\varepsilon}=\left(u_{\varepsilon}^{a}, u_{\varepsilon}^{b}\right)$ satisfying (3.4). Let $\mathcal{V}$ be defined in (2.10). Then, there exists a subsequence of $\{\varepsilon\}$, still denoted by $\{\varepsilon\}$, and $\left(u^{a}, u^{b}\right) \in \mathcal{V}$ (in possible dependence on the subsequence) such that

$$
\begin{cases}u_{\varepsilon}^{a} \rightarrow u^{a} \text { weakly in } H^{1}\left(\Omega^{a}\right), & \frac{1}{\varepsilon} \partial_{x_{1}} u_{\varepsilon}^{a} \rightarrow 0, \text { weakly in } L^{2}\left(\Omega^{a}\right), \\ u_{\varepsilon}^{b} \rightarrow u^{b} \text { weakly in } H^{1}\left(\Omega^{b}\right), & \frac{1}{\varepsilon} \partial_{x_{2}} u_{\varepsilon}^{b} \rightarrow 0, \text { weakly in } L^{2}\left(\Omega^{b}\right),\end{cases}
$$

as $\varepsilon$ tends to zero. Moreover, $\left(u^{a}, u^{b}\right)$ satisfies (2.9) and

$$
|\omega| \int_{0}^{d}\left|u^{a}\right|^{2} d x_{2}+\int_{\omega}\left|u^{b}\right|^{2} d x_{1}=1
$$


Proof. The normalized condition (3.4) implies that there exists a subsequence of $\{\varepsilon\}$, still denoted by $\{\varepsilon\}$, and $\left(u^{a}, u^{b}\right) \in L^{2}\left(\Omega^{a}\right) \times L^{2}\left(\Omega^{b}\right)$ (in possible dependence on the subsequence) such that

$$
u_{\varepsilon}^{a} \rightarrow u^{a} \text { weakly in } L^{2}\left(\Omega^{a}\right), \quad u_{\varepsilon}^{b} \rightarrow u^{b} \text { weakly in } L^{2}\left(\Omega^{b}\right),
$$

as $\varepsilon$ tends to zero. Then, the result is obtained adapting the proof of Theorem 1.1 in [13] with $f_{\varepsilon}=\lambda_{\varepsilon}\left(u_{\varepsilon}^{a}, u_{\varepsilon}^{b}\right)$ and $f=\mu\left(u^{a}, u^{b}\right)$ (take also into account Remark 1.4 in [13]).

Remark 4.8. Proposition 4.7 agrees with Theorem 2.1. In fact, if $\left\{\lambda_{\varepsilon, k}\right\}_{k \in \mathbb{N}}$ are the eigenvalues of (2.6) and $\left\{u_{\varepsilon, k}\right\}_{k \in \mathbb{N}}$ are the corresponding eigenfunctions such that $\left\|u_{\varepsilon, k}\right\|_{L^{2}\left(\Omega^{a} \cup \Omega^{b}\right)}=$ 1, then, by Theorem 2.1, for each fixed $k \in \mathbb{N}$, the sequence $\lambda_{\varepsilon, k}$ converges, when $\varepsilon$ tends to zero, towards $\mu_{k}$, the $k$-th eigenvalue of (2.9). Moreover, there exists a subsequence of $\{\varepsilon\}$, still denoted by $\{\varepsilon\}$, and a sequence $\left\{u_{k}^{a}, u_{k}^{b}\right\}_{k \in \mathbb{N}} \subset \mathcal{V}, \mathcal{V}$ defined by (2.10), such that

$$
\begin{aligned}
& \lim _{\varepsilon \rightarrow 0}\left(\left\|u_{\varepsilon, k}^{a}-u_{k}^{a}\right\|_{L^{2}\left(\Omega^{a}\right)}+\left\|\partial_{x_{1}} u_{\varepsilon, k}^{a}\right\|_{L^{2}\left(\Omega^{a}\right)}+\left\|\partial_{x_{2}}\left(u_{\varepsilon, k}^{a}-u_{k}^{a}\right)\right\|_{L^{2}\left(\Omega^{a}\right)}\right)=0, \\
& \lim _{\varepsilon \rightarrow 0}\left(\left\|u_{\varepsilon, k}^{b}-u_{k}^{b}\right\|_{L^{2}\left(\Omega^{b}\right)}+\left\|\partial_{x_{1}}\left(u_{\varepsilon, k}^{b}-u_{k}^{b}\right)\right\|_{L^{2}\left(\Omega^{b}\right)}+\left\|\partial_{x_{2}} u_{\varepsilon, k}^{b}\right\|_{L^{2}\left(\Omega^{b}\right)}\right)=0
\end{aligned}
$$

for every $k \in \mathbb{N}$, and $u_{k}=\left(u_{k}^{a}, u_{k}^{b}\right)$ is an eigenfunction of problem (2.9) with eigenvalue $\mu_{k}$. Note that in Theorem 2.1 a different convergence result on the limit eigenfunctions appears. It depends on the different initial normalization of the eigenfunctions.

\section{The main results}

In this section, we characterize the behavior of the eigenfunctions associated with eigenvalues $\lambda_{\varepsilon}$ of $(2.6)$ of order $O\left(\varepsilon^{-2}\right)$ (see Theorem 5.6). We also provide bounds for the convergence rate of the values $\varepsilon^{2} \lambda_{\varepsilon}$ near an eigenvalue of (3.7) and their corresponding eigenfunctions (see Theorem 5.2, Theorem 5.3, and Theorem 5.4). These results justify and complement those in Section 4.1.

First, we introduce here some notations that will be used throughout the section.

Fix $\varepsilon>0$, let $V_{\varepsilon}$ be the space defined in (2.7) equipped with the following inner product

$$
\begin{aligned}
& (\cdot, \cdot)_{\varepsilon}:(v, w)=\left(\left(v^{a}, v^{b}\right),\left(w^{a}, w^{b}\right)\right) \in V_{\varepsilon}^{2} \longrightarrow(v, w)_{\varepsilon} \\
& =\int_{\Omega^{a}}\left(v^{a} w^{a}+\partial_{x_{1}} v^{a} \partial_{x_{1}} w^{a}+\varepsilon^{2} \partial_{x_{2}} v^{a} \partial_{x_{2}} w^{a}\right) d x \\
& +\int_{\Omega^{b}}\left(v^{b} w^{b}+\varepsilon^{2} \partial_{x_{1}} v^{b} \partial_{x_{1}} w^{b}+\partial_{x_{2}} v^{b} \partial_{x_{2}} w^{b}\right) d x .
\end{aligned}
$$

Note that, in order to obtain bounds for the eigenfunctions associated with the eigenvalues of $(2.6)$ of order $O\left(\varepsilon^{-2}\right)$, it is equivalent to normalize either with $(\cdot, \cdot)_{\varepsilon}^{\frac{1}{2}}$ or $\|$. $\|_{L^{2}\left(\Omega^{a} \cup \Omega^{b}\right)}$ since $\varepsilon^{2} \lambda_{\varepsilon}=O(1)$ and

$$
\left(u_{\varepsilon}, u_{\varepsilon}\right)_{\varepsilon}=\left(\varepsilon^{2} \lambda_{\varepsilon}+1\right)\left(\left\|u_{\varepsilon}^{a}\right\|_{L^{2}\left(\Omega^{a}\right)}^{2}+\left\|u_{\varepsilon}^{b}\right\|_{L^{2}\left(\Omega^{b}\right)}^{2}\right) .
$$


Let

$$
B_{\varepsilon}: w=\left(w^{a}, w^{b}\right) \in V_{\varepsilon} \longrightarrow B_{\varepsilon}(w) \in V_{\varepsilon}
$$

be the operator defined by

$$
\left\{\begin{array}{l}
B_{\varepsilon}(w) \in V_{\varepsilon}, \\
\left(B_{\varepsilon}(w), v\right)_{\varepsilon}=\int_{\Omega^{a}} w^{a} v^{a} d x+\int_{\Omega^{b}} w^{b} v^{b} d x, \quad \forall v=\left(v^{a}, v^{b}\right) \in V_{\varepsilon} .
\end{array}\right.
$$

This operator is linear, self-adjoint, positive, and compact. Consequently, $B_{\varepsilon}$ possesses a countable infinity of positive eigenvalues converging to zero (for instance, see Th. 4.6 in $[42])$

$$
\rho_{\varepsilon, 1} \geq \rho_{\varepsilon, 2} \geq \cdots \rho_{\varepsilon, k} \geq \cdots \rightarrow 0 .
$$

Moreover, it is easily verified that

$$
\rho_{\varepsilon, k}=\frac{1}{\varepsilon^{2} \lambda_{\varepsilon, k}+1}, \quad \forall k \in \mathbb{N}
$$

where $\left\{\lambda_{\varepsilon, k}\right\}_{k \in \mathbb{N}}$ is the sequence of the eigenvalues of (2.6).

We also introduce here a result which will be used in what follows (see Section III.1 in [38] for its proof):

Lemma 5.1. Let $A: H \longrightarrow H$ be a linear, self-adjoint, positive and compact operator on a Hilbert space $H$. Let $u \in H$, with $\|u\|_{H}=1$ and $\lambda, r>0$ such that $\|A u-\lambda u\|_{H}<r$. Then, there exists an eigenvalue $\lambda_{i}$ of $A$ satisfying $\left|\lambda-\lambda_{i}\right|<r$. Moreover, for any $r^{*}>r$ there is $u^{*} \in H$, with $\left\|u^{*}\right\|_{H}=1$, $u^{*}$ being a linear combination of eigenfunctions associated with all the eigenvalues of operator $A$ lying on the segment $\left[\lambda-r^{*}, \lambda+r^{*}\right]$, such that

$$
\left\|u-u^{*}\right\|_{H}<\frac{2 r}{r^{*}}
$$

Theorem 5.2. Let $\lambda^{a}$ be an eigenvalue of (1.3). Then, for each $\varepsilon$ there exists an eigenvalue $\lambda_{\varepsilon}$ of (2.6) such that

$$
\left|\varepsilon^{2} \lambda_{\varepsilon}-\lambda^{a}\right| \leq c_{1} \varepsilon^{\frac{3}{2}}
$$

where $c_{1}$ is a constant independent of $\varepsilon$.

Let $z^{a}$ be an eigenfunction of (1.3) associated with $\lambda^{a}$ such that

$$
\left\|z^{a}\right\|_{L^{2}(\omega)}=1 .
$$

Let $(\mu, \alpha)$ be an eigenelement of (1.6) such that

$$
\|\alpha\|_{L^{2}(] 0, d[)}=1 .
$$

Set

$$
u^{a}\left(x_{1}, x_{2}\right)=z^{a}\left(x_{1}\right) \alpha\left(x_{2}\right) \text {, in } \Omega^{a} .
$$


Let $\theta \in] 0, \frac{3}{2}\left[\right.$. Then, there exists $\left.K_{a} \in\right] 0,+\infty[$ and for each $\varepsilon$, there exists a linear combination $\widetilde{u_{\varepsilon}}=\left(\widetilde{u_{\varepsilon}^{a}}, \widetilde{u_{\varepsilon}^{b}}\right) \in V_{\varepsilon}$ of eigenfunctions of (2.6) associated with all the eigenvalues $\lambda_{\varepsilon, l(\varepsilon)}, \cdots, \lambda_{\varepsilon, l(\varepsilon)+\nu(\varepsilon)}, l(\varepsilon) \in \mathbb{N}, \nu(\varepsilon) \in \mathbb{N}_{0}$, satisfying

$$
\varepsilon^{2} \lambda_{\varepsilon, l(\varepsilon)}, \cdots, \varepsilon^{2} \lambda_{\varepsilon, l(\varepsilon)+\nu(\varepsilon)} \in\left[\lambda^{a}-K_{a} \varepsilon^{\theta}, \lambda^{a}+K_{a} \varepsilon^{\theta}\right]
$$

such that

$$
\begin{gathered}
\left(\widetilde{u_{\varepsilon}}, \widetilde{u}_{\varepsilon}\right)_{\varepsilon}=1, \\
\left\|\widetilde{u_{\varepsilon}^{a}}-\delta_{\varepsilon}^{a} u^{a}\right\|_{L^{2}\left(\Omega^{a}\right)}+\left\|\partial_{x_{1}}\left(\widetilde{u_{\varepsilon}^{a}}-\delta_{\varepsilon}^{a} u^{a}\right)\right\|_{L^{2}\left(\Omega^{a}\right)}+\varepsilon\left\|\partial_{x_{2}}\left(\widetilde{u_{\varepsilon}^{a}}-\delta_{\varepsilon}^{a} u^{a}\right)\right\|_{L^{2}\left(\Omega^{a}\right)} \\
+\left\|\widetilde{u_{\varepsilon}^{b}}\right\|_{L^{2}\left(\Omega^{b}\right)}+\varepsilon\left\|\partial_{x_{1}} \widetilde{u_{\varepsilon}^{b}}\right\|_{L^{2}\left(\Omega^{b}\right)}+\left\|\partial_{x_{2}} \widetilde{u_{\varepsilon}^{b}}\right\|_{L^{2}\left(\Omega^{b}\right)} \leq c_{2} \varepsilon^{\frac{3}{2}-\theta},
\end{gathered}
$$

where $c_{2}$ is a constant independent of $\varepsilon$ and $\left.\left\{\delta_{\varepsilon}^{a}\right\}_{\varepsilon} \subset\right] 0,+\infty\left[\right.$ is such that $\lim _{\varepsilon \rightarrow 0} \delta_{\varepsilon}^{a}=\frac{1}{\sqrt{1+\lambda^{a}}}$.

Proof. We apply Lemma 5.1 for parameters $\lambda$ and test functions $u$ that we construct taking into account Proposition 3.4 and Proposition 4.5.

Let

$$
v_{\varepsilon}^{a}\left(x_{1}, x_{2}\right)=\left\{\begin{array}{l}
\left.z^{a}\left(x_{1}\right) \alpha\left(x_{2}\right) \text { in } \omega \times\right] \varepsilon, d[, \\
\left.z^{a}\left(x_{1}\right) \alpha(\varepsilon) \frac{x_{2}}{\varepsilon}, \text { in } \omega \times\right] 0, \varepsilon[.
\end{array}\right.
$$

We observe that $v_{\varepsilon}=\left(v_{\varepsilon}^{a}, 0\right) \in V_{\varepsilon}$.

The first step is devoted to proving that

$$
\begin{aligned}
& \left.\exists c_{3} \in\right] 0,+\infty\left[:\left(\lambda^{a}+\varepsilon^{2} \mu+1\right)\left(B_{\varepsilon}\left(v_{\varepsilon}\right)-\frac{1}{\lambda^{a}+\varepsilon^{2} \mu+1} v_{\varepsilon}, v\right)_{\varepsilon}\right. \\
& \leq c_{3} \varepsilon^{\frac{3}{2}}(v, v)_{\varepsilon}^{\frac{1}{2}}, \quad \forall v \in V_{\varepsilon} .
\end{aligned}
$$

Let us note that

$$
\begin{aligned}
& \left(\lambda^{a}+\varepsilon^{2} \mu+1\right)\left(B_{\varepsilon}\left(v_{\varepsilon}\right)-\frac{1}{\lambda^{a}+\varepsilon^{2} \mu+1} v_{\varepsilon}, v\right)_{\varepsilon} \\
& =\left(\lambda^{a}+\varepsilon^{2} \mu\right) \int_{\Omega^{a}} v_{\varepsilon}^{a} v^{a} d x-\int_{\Omega^{a}}\left(\partial_{x_{1}} v_{\varepsilon}^{a} \partial_{x_{1}} v^{a}+\varepsilon^{2} \partial_{x_{2}} v_{\varepsilon}^{a} \partial_{x_{2}} v^{a}\right) d x, \\
& \forall v=\left(v^{a}, v^{b}\right) \in V_{\varepsilon} .
\end{aligned}
$$

As far as the first and the third terms in right-hand side of (5.8) are concerned, since 
$z^{a}$ is an eigenfunction of (1.3) associated with $\lambda^{a}$, one has

$$
\begin{aligned}
& \lambda^{a} \int_{\Omega^{a}} v_{\varepsilon}^{a} v^{a} d x-\int_{\Omega^{a}} \partial_{x_{1}} v_{\varepsilon}^{a} \partial_{x_{1}} v^{a} d x \\
& =\int_{0}^{\varepsilon}\left(\alpha(\varepsilon) \frac{x_{2}}{\varepsilon} \int_{\omega}\left(\lambda^{a} z^{a}\left(x_{1}\right) v^{a}\left(x_{1}, x_{2}\right)-\left(z^{a}\right)^{\prime}\left(x_{1}\right) \partial_{x_{1}} v^{a}\left(x_{1}, x_{2}\right)\right) d x_{1}\right) d x_{2} \\
& =\int_{\varepsilon}^{d}\left(\alpha\left(x_{2}\right) \int_{\omega}\left(\lambda^{a} z^{a}\left(x_{1}\right) v^{a}\left(x_{1}, x_{2}\right)-\left(z^{a}\right)^{\prime}\left(x_{1}\right) \partial_{x_{1}} v^{a}\left(x_{1}, x_{2}\right)\right) d x_{1}\right) d x_{2}=0, \\
& \forall v=\left(v^{a}, v^{b}\right) \in V_{\varepsilon} .
\end{aligned}
$$

For the other terms in right-hand side of (5.8), we have

$$
\begin{aligned}
& \varepsilon^{2} \mu \int_{\Omega^{a}} v_{\varepsilon}^{a} v^{a} d x-\int_{\Omega^{a}} \varepsilon^{2} \partial_{x_{2}} v_{\varepsilon}^{a} \partial_{x_{2}} v^{a} d x \\
& =\varepsilon^{2} \int_{\omega} z^{a}\left(x_{1}\right)\left(\int_{0}^{d}\left(\mu \alpha\left(x_{2}\right) v^{a}\left(x_{1}, x_{2}\right)-\alpha^{\prime}\left(x_{2}\right) \partial_{x_{2}} v^{a}\left(x_{1}, x_{2}\right)\right) d x_{2}\right) d x_{1} \\
& +\varepsilon^{2} \int_{\omega} z^{a}\left(x_{1}\right)\left(\int_{0}^{\varepsilon} \mu\left(\alpha(\varepsilon) \frac{x_{2}}{\varepsilon}-\alpha\left(x_{2}\right)\right) v^{a}\left(x_{1}, x_{2}\right) d x_{2}\right) d x_{1} \\
& -\varepsilon^{2} \int_{\omega} z^{a}\left(x_{1}\right)\left(\int_{0}^{\varepsilon}\left(\alpha(\varepsilon) \frac{1}{\varepsilon}-\alpha^{\prime}\left(x_{2}\right)\right) \partial_{x_{2}} v^{a}\left(x_{1}, x_{2}\right) d x_{2}\right) d x_{1} \\
& \forall v=\left(v^{a}, v^{b}\right) \in V_{\varepsilon} .
\end{aligned}
$$

As far as the first integral in the right-hand side of (5.10) is concerned, taking into account that $(\mu, \alpha)$ is an eigenelement of (1.6) and that

$$
v^{a}\left(x_{1}, 0\right)=v^{b}\left(\varepsilon x_{1}, 0\right), \text { on } \omega
$$

one easily obtains

$$
\begin{aligned}
& \varepsilon^{2} \int_{\omega} z^{a}\left(x_{1}\right)\left(\int_{0}^{d}\left(\mu \alpha\left(x_{2}\right) v^{a}\left(x_{1}, x_{2}\right)-\alpha^{\prime}\left(x_{2}\right) \partial_{x_{2}} v^{a}\left(x_{1}, x_{2}\right)\right) d x_{2}\right) d x_{1} \\
& =\varepsilon^{2} \alpha^{\prime}(0) \int_{\omega} z^{a}\left(x_{1}\right) v^{a}\left(x_{1}, 0\right) d x_{1}=\varepsilon^{2} \alpha^{\prime}(0) \int_{\omega} z^{a}\left(x_{1}\right) v^{b}\left(\varepsilon x_{1}, 0\right) d x_{1} \\
& \leq \varepsilon^{2}\left|\alpha^{\prime}(0)\right|\left(\int_{\omega}\left|v^{b}\left(\varepsilon x_{1}, 0\right)\right|^{2} d x_{1}\right)^{\frac{1}{2}} \leq \varepsilon^{2}\left|\alpha^{\prime}(0)\right| \varepsilon^{-\frac{1}{2}}\left\|v^{b}(\cdot, 0)\right\|_{L^{2}(\omega)} \\
& \leq \varepsilon^{\frac{3}{2}}\left|\alpha^{\prime}(0)\right| 2\left(\left\|v^{b}\right\|_{L^{2}\left(\Omega^{b}\right)}^{2}+\left\|\partial_{x_{2}} v^{b}\right\|_{L^{2}\left(\Omega^{b}\right)}^{2}\right)^{\frac{1}{2}} \leq \varepsilon^{\frac{3}{2}}\left|\alpha^{\prime}(0)\right| 2(v, v)_{\varepsilon}^{\frac{1}{2}} \\
& \forall v=\left(v^{a}, v^{b}\right) \in V_{\varepsilon} .
\end{aligned}
$$


As far as the second integral in the right-hand side of (5.10) is concerned, thanks to the smoothness of the functions $z^{a}$, $\alpha$, i.e. $z^{a} \in C^{\infty}(\bar{\omega})$ and $\alpha \in C^{\infty}([0, d])$,

$$
\begin{aligned}
& \left.\exists c_{4} \in\right] 0,+\infty\left[: \varepsilon^{2} \int_{\omega} z^{a}\left(x_{1}\right)\left(\int_{0}^{\varepsilon} \mu\left(\alpha(\varepsilon) \frac{x_{2}}{\varepsilon}-\alpha\left(x_{2}\right)\right) v^{a}\left(x_{1}, x_{2}\right) d x_{2}\right) d x_{1}\right. \\
& \leq c_{4} \varepsilon^{2}(v, v)_{\varepsilon}^{\frac{1}{2}}, \quad \forall v=\left(v^{a}, v^{b}\right) \in V_{\varepsilon} .
\end{aligned}
$$

As far as the last integral in the right-hand side of (5.10) is concerned, at first remark that the condition $\alpha(0)=0$ (see (1.6)) implies that

$$
|\alpha(\varepsilon)| \leq\left\|\alpha^{\prime}\right\|_{L^{\infty}([0, d])}|\varepsilon|, \quad \forall \varepsilon .
$$

Consequently,

$$
\begin{aligned}
& \left.\exists c_{5} \in\right] 0,+\infty\left[: \varepsilon^{2} \int_{\omega} z^{a}\left(x_{1}\right)\left(\int_{0}^{\varepsilon}\left(\alpha(\varepsilon) \frac{1}{\varepsilon}-\alpha^{\prime}\left(x_{2}\right)\right) \partial_{x_{2}} v^{a}\left(x_{1}, x_{2}\right) d x_{2}\right) d x_{1}\right. \\
& \leq c_{5} \varepsilon^{2} \varepsilon^{\frac{1}{2}}\left\|\partial_{x_{2}} v^{a}\right\|_{L^{2}\left(\Omega^{a}\right)}=c_{5} \varepsilon^{\frac{3}{2}}(v, v)_{\varepsilon}^{\frac{1}{2}}, \quad \forall v=\left(v^{a}, v^{b}\right) \in V_{\varepsilon} .
\end{aligned}
$$

Finally (5.7) follows from (5.8), (5.9), (5.10), (5.11), (5.12), and (5.14). Choosing

$$
v=B_{\varepsilon}\left(v_{\varepsilon}\right)-\frac{1}{\lambda^{a}+\varepsilon^{2} \mu+1} v_{\varepsilon}
$$

in (5.7) gives

$$
\begin{aligned}
& \left.\exists c_{3} \in\right] 0,+\infty\left[:\left(B_{\varepsilon}\left(v_{\varepsilon}\right)-\frac{1}{\lambda^{a}+\varepsilon^{2} \mu+1} v_{\varepsilon}, B_{\varepsilon}\left(v_{\varepsilon}\right)-\frac{1}{\lambda^{a}+\varepsilon^{2} \mu+1} v_{\varepsilon}\right)_{\varepsilon}\right. \\
& \leq c_{3} \varepsilon^{\frac{3}{2}} \frac{1}{\lambda^{a}+\varepsilon^{2} \mu+1}\left(B_{\varepsilon}\left(v_{\varepsilon}\right)-\frac{1}{\lambda^{a}+\varepsilon^{2} \mu+1} v_{\varepsilon}, B_{\varepsilon}\left(v_{\varepsilon}\right)-\frac{1}{\lambda^{a}+\varepsilon^{2} \mu+1} v_{\varepsilon}\right)_{\varepsilon}^{\frac{1}{2}},
\end{aligned}
$$

i.e.

$$
\begin{aligned}
& \left.\exists c_{6} \in\right] 0,+\infty[: \\
& \left(B_{\varepsilon}\left(v_{\varepsilon}\right)-\frac{1}{\lambda^{a}+\varepsilon^{2} \mu+1} v_{\varepsilon}, B_{\varepsilon}\left(v_{\varepsilon}\right)-\frac{1}{\lambda^{a}+\varepsilon^{2} \mu+1} v_{\varepsilon}\right)_{\varepsilon}^{\frac{1}{2}} \leq c_{6} \varepsilon^{\frac{3}{2}} .
\end{aligned}
$$

On the other hand, using that $z^{a}$ is an eigenfunction of (1.3) associated with $\lambda^{a}$ and satisfies (5.3), and that $\alpha$ satisfies (5.4), and $\alpha(0)=0$, it is easy to show that

$$
\lim _{\varepsilon \rightarrow 0}\left(v_{\varepsilon}, v_{\varepsilon}\right)_{\varepsilon}=1+\lambda^{a} .
$$

Combining (5.15) and (5.16) gives

$$
\begin{aligned}
& \left.\exists c_{7} \in\right] 0,+\infty[: \\
& \left(B_{\varepsilon}\left(\delta_{\varepsilon}^{a} v_{\varepsilon}\right)-\frac{1}{\lambda^{a}+\varepsilon^{2} \mu+1} \delta_{\varepsilon}^{a} v_{\varepsilon}, B_{\varepsilon}\left(\delta_{\varepsilon}^{a} v_{\varepsilon}\right)-\frac{1}{\lambda^{a}+\varepsilon^{2} \mu+1} \delta_{\varepsilon}^{a} v_{\varepsilon}\right)_{\varepsilon}^{\frac{1}{2}} \leq c_{7} \varepsilon^{\frac{3}{2}} .
\end{aligned}
$$


with

$$
\delta_{\varepsilon}^{a}=\frac{1}{\left(v_{\varepsilon}, v_{\varepsilon}\right)_{\varepsilon}^{\frac{1}{2}}} .
$$

Applying Lemma 5.1 with $A=B_{\varepsilon}, \lambda=\left(\lambda^{a}+\varepsilon^{2} \mu+1\right)^{-1}$ and $u=\left(\delta_{\varepsilon}^{a} v_{\varepsilon}^{a}, 0\right)$, where $v_{\varepsilon}^{a}$ and $\delta_{\varepsilon}^{a}$ are defined in (5.6) and (5.18), respectively, and taking into account (5.1), from (5.17) one derives that

$$
\left|\frac{1}{\varepsilon^{2} \lambda_{\varepsilon}+1}-\frac{1}{\lambda^{a}+\varepsilon^{2} \mu+1}\right| \leq c_{7} \varepsilon^{\frac{3}{2}},
$$

which proves (5.2). Finally, applying Lemma 5.1 with $r^{*}=C \varepsilon^{\theta}$, for any positive constant $C$, one completes the proof of this theorem.

Theorem 5.3. Let $\lambda^{b}$ be an eigenvalue of (1.4). Then, for each $\varepsilon$ there exists an eigenvalue $\lambda_{\varepsilon}$ of (2.6) such that

$$
\left|\varepsilon^{2} \lambda_{\varepsilon}-\lambda^{b}\right| \leq c_{1} \varepsilon^{\frac{3}{2}}
$$

where $c_{1}$ is a constant independent of $\varepsilon$.

Let $z^{b}$ be an eigenfunction of (1.4) associated with $\lambda^{b}$ such that

$$
\left\|z^{b}\right\|_{L^{2}(]-1,0[)}=1 .
$$

Let $(\mu, \beta)$ be an eigenelement of (1.7) such that

$$
\|\beta\|_{L^{2}(\omega)}=1 .
$$

Set

$$
u^{b}\left(x_{1}, x_{2}\right)=z^{b}\left(x_{2}\right) \beta\left(x_{1}\right), \text { in } \Omega^{b} .
$$

Let $\theta \in] 0, \frac{3}{2}\left[\right.$. Then, there exists $\left.K_{b} \in\right] 0,+\infty[$ and for each $\varepsilon$, there exists a linear combination $\widetilde{u_{\varepsilon}}=\left(\widetilde{u_{\varepsilon}^{a}}, \widetilde{u_{\varepsilon}^{b}}\right) \in V_{\varepsilon}$ of eigenfunctions of (2.6) associated with all the eigenvalues $\lambda_{\varepsilon, l(\varepsilon)}, \cdots, \lambda_{\varepsilon, l(\varepsilon)+\nu(\varepsilon)}, l(\varepsilon) \in \mathbb{N}, \nu(\varepsilon) \in \mathbb{N}_{0}$, satisfying

$$
\varepsilon^{2} \lambda_{\varepsilon, l(\varepsilon)}, \cdots, \varepsilon^{2} \lambda_{\varepsilon, l(\varepsilon)+\nu(\varepsilon)} \in\left[\lambda^{b}-K_{b} \varepsilon^{\theta}, \lambda^{b}+K_{b} \varepsilon^{\theta}\right]
$$

such that

$$
\begin{gathered}
\left(\widetilde{u_{\varepsilon}}, \widetilde{u_{\varepsilon}}\right)_{\varepsilon}=1, \\
\left\|\widetilde{u_{\varepsilon}^{a}}\right\|_{L^{2}\left(\Omega^{a}\right)}+\left\|\partial_{x_{1}} \widetilde{u_{\varepsilon}^{a}}\right\|_{L^{2}\left(\Omega^{a}\right)}+\varepsilon\left\|\partial_{x_{2}} \widetilde{u_{\varepsilon}^{a}}\right\|_{L^{2}\left(\Omega^{a}\right)}+\left\|\widetilde{u_{\varepsilon}^{b}}-\delta_{\varepsilon}^{b} u^{b}\right\|_{L^{2}\left(\Omega^{b}\right)} \\
+\varepsilon\left\|\partial_{x_{1}}\left(\widetilde{u_{\varepsilon}^{b}}-\delta_{\varepsilon}^{b} u^{b}\right)\right\|_{L^{2}\left(\Omega^{b}\right)}+\left\|\partial_{x_{2}}\left(\widetilde{u_{\varepsilon}^{b}}-\delta_{\varepsilon}^{b} u^{b}\right)\right\|_{L^{2}\left(\Omega^{b}\right)} \leq c_{2} \varepsilon^{\frac{3}{2}-\theta},
\end{gathered}
$$

where $c_{2}$ is a constant independent of $\varepsilon$ and $\left.\left\{\delta_{\varepsilon}^{b}\right\}_{\varepsilon} \subset\right] 0,+\infty\left[\right.$ is such that $\lim _{\varepsilon \rightarrow 0} \delta_{\varepsilon}^{b}=\frac{1}{\sqrt{1+\lambda^{b}}}$.

Proof. Similar arguments to the proof of Theorem 5.2 allow us to prove this theorem. Now, in order to apply Lemma 5.1 the function $v_{\varepsilon}$ used is

$$
v_{\varepsilon}\left(x_{1}, x_{2}\right)=\left\{\begin{array}{l}
0, \text { in } \omega \times] \varepsilon, d[, \\
\left.z^{b}(0) \beta\left(\varepsilon x_{1}\right) \frac{\varepsilon-x_{2}}{\varepsilon}, \text { in } \omega \times\right] 0, \varepsilon[, \\
z^{b}\left(x_{2}\right) \beta\left(x_{1}\right), \text { in } \Omega^{b},
\end{array}\right.
$$


where $z^{b}$ and $\beta$ are defined in the statement of the theorem (cf. Proposition 3.4 and Proposition 4.5).

Theorem 5.4. Let $\left(\lambda^{a}, z^{a}\right)$ and $\left(\lambda^{b}, z^{b}\right)$ be eigenelements of (1.3) and (1.4), respectively, such that $\lambda^{a}=\lambda^{b}=\lambda$ and

$$
\left\|z^{a}\right\|_{L^{2}(\omega)}=1=\left\|z^{b}\right\|_{L^{2}(]-1,0[)} .
$$

Let $\left(\mu^{a}, \alpha\right)$ and $\left(\mu^{b}, \beta\right)$ be eigenelements of (1.6) and (1.7), respectively, such that

$$
\|\alpha\|_{L^{2}(] 0, d[)}=1=\|\beta\|_{L^{2}(\omega)} .
$$

Set

$$
\begin{aligned}
& u^{a}\left(x_{1}, x_{2}\right)=z^{a}\left(x_{1}\right) \alpha\left(x_{2}\right), \text { in } \Omega^{a}, \\
& u^{b}\left(x_{1}, x_{2}\right)=z^{b}\left(x_{2}\right) \beta\left(x_{1}\right), \text { in } \Omega^{b} .
\end{aligned}
$$

Let $\theta \in] 0, \frac{3}{2}[$. Then, there exists $K \in] 0,+\infty[$ and for each $\varepsilon$, there exists a linear combination $\widetilde{u_{\varepsilon}}=\left(\widetilde{u_{\varepsilon}^{a}}, \widetilde{u_{\varepsilon}^{b}}\right) \in V_{\varepsilon}$ of eigenfunctions of (2.6) associated with all the eigenvalues $\lambda_{\varepsilon, l(\varepsilon)}, \cdots, \lambda_{\varepsilon, l(\varepsilon)+\nu(\varepsilon)}, l(\varepsilon) \in \mathbb{N}, \nu(\varepsilon) \in \mathbb{N}_{0}$, satisfying

$$
\varepsilon^{2} \lambda_{\varepsilon, l(\varepsilon)}, \cdots, \varepsilon^{2} \lambda_{\varepsilon, l(\varepsilon)+\nu(\varepsilon)} \in\left[\lambda-K \varepsilon^{\theta}, \lambda+K \varepsilon^{\theta}\right]
$$

such that

$$
\begin{aligned}
& \left(\widetilde{u_{\varepsilon}}, \widetilde{u_{\varepsilon}}\right)_{\varepsilon}=1, \\
& \left\|\widetilde{u_{\varepsilon}^{a}}-\delta_{\varepsilon}^{a} u^{a}\right\|_{L^{2}\left(\Omega^{a}\right)}+\left\|\partial_{x_{1}}\left(\widetilde{u_{\varepsilon}^{a}}-\delta_{\varepsilon}^{a} u^{a}\right)\right\|_{L^{2}\left(\Omega^{a}\right)}+\varepsilon\left\|\partial_{x_{2}}\left(\widetilde{u_{\varepsilon}^{a}}-\delta_{\varepsilon}^{a} u^{a}\right)\right\|_{L^{2}\left(\Omega^{a}\right)} \\
& +\left\|\widetilde{u_{\varepsilon}^{b}}-\delta_{\varepsilon}^{b} u^{b}\right\|_{L^{2}\left(\Omega^{b}\right)}+\varepsilon\left\|\partial_{x_{1}}\left(\widetilde{u_{\varepsilon}^{b}}-\delta_{\varepsilon}^{b} u^{b}\right)\right\|_{L^{2}\left(\Omega^{b}\right)}+\left\|\partial_{x_{2}}\left(\widetilde{u_{\varepsilon}^{b}}-\delta_{\varepsilon}^{b} u^{b}\right)\right\|_{L^{2}\left(\Omega^{b}\right)} \\
& \leq c_{2} \varepsilon^{\frac{3}{2}-\theta}
\end{aligned}
$$

where $c_{2}$ is a constant independent of $\varepsilon$ and $\left.\left\{\delta_{\varepsilon}^{a}\right\}_{\varepsilon} \cup\left\{\delta_{\varepsilon}^{b}\right\}_{\varepsilon} \subset\right] 0,+\infty\left[\right.$ is such that $\lim _{\varepsilon \rightarrow 0} \delta_{\varepsilon}^{a}=$ $\frac{1}{\sqrt{2(1+\lambda)}}=\lim _{\varepsilon \rightarrow 0} \delta_{\varepsilon}^{b}$

Proof. This result is a direct consequence of Theorem 5.2 and Theorem 5.3.

Remark 5.5. Note that the values $\mu^{a}$ and $\mu^{b}$ in Theorem 5.4 can be different. In case $\mu^{a}=\mu^{b}$, there exists a linear combination $\widetilde{u_{\varepsilon}}$ satisfying the statement of Theorem 5.4 where $\delta_{\varepsilon}^{a}=\delta_{\varepsilon}^{b}$. To prove it, we apply Lemma 5.1 with $\lambda=\left(\lambda^{a}+\varepsilon^{2} \mu^{a}+1\right)^{-1}$ and $u=\delta_{\varepsilon}^{a} v_{\varepsilon}$ where the function $v_{\varepsilon}$ is defined by

$$
v_{\varepsilon}\left(x_{1}, x_{2}\right)=\left\{\begin{array}{l}
\left.z^{a}\left(x_{1}\right) \alpha\left(x_{2}\right), \text { in } \omega \times\right] \varepsilon, d[, \\
\left.z^{a}\left(x_{1}\right) \alpha(\varepsilon) \frac{x_{2}}{\varepsilon}+z^{b}(0) \beta\left(\varepsilon x_{1}\right) \frac{\varepsilon-x_{2}}{\varepsilon}, \text { in } \omega \times\right] 0, \varepsilon[, \\
z^{b}\left(x_{2}\right) \beta\left(x_{1}\right), \text { in } \Omega^{b},
\end{array}\right.
$$


and $\delta_{\varepsilon}^{a}=\left(v_{\varepsilon}, v_{\varepsilon}\right)_{\varepsilon}^{-\frac{1}{2}}$, being $z^{a}, z^{b}, \alpha$ and $\beta$ the functions defined in the statement of the theorem (cf. the proof of Theorem 5.2).

On the other hand, since $\alpha(0)=0$, one can choose

$$
\left.v_{\varepsilon}\left(x_{1}, x_{2}\right)=z^{a}\left(x_{1}\right) \alpha\left(x_{2}\right)+z^{b}(0) \beta\left(\varepsilon x_{1}\right) \frac{\varepsilon-x_{2}}{\varepsilon}, \text { in } \omega \times\right] 0, \varepsilon[
$$

and

$$
v_{\varepsilon}^{a}\left(x_{1}, x_{2}\right)=z^{a}\left(x_{1}\right) \alpha\left(x_{2}\right) \text { in } \Omega^{a},
$$

instead of (5.19) $)_{2}$ and (5.6), respectively, getting the same estimates. We use (5.19) and (5.6) to unify proofs (cf. Proposition 3.4, Theorem 5.2, Theorem 5.3 and Theorem 5.4).

Theorem 5.6. Let $\lambda \in] 0,+\infty[$.

1. There exists a subsequence of $\varepsilon$ converging towards zero, still denoted by $\varepsilon$, and a vanishing sequence of positive numbers $\left\{d_{\varepsilon}\right\}_{\varepsilon}$, such that $\varepsilon^{2} \lambda_{\varepsilon, k(\varepsilon)} \in\left[\lambda-d_{\varepsilon}, \lambda+d_{\varepsilon}\right]$, for some eigenvalue $\lambda_{\varepsilon, k(\varepsilon)}$ of (2.6).

2. $\lambda$ is an eigenvalue of (3.7) iff there is a vanishing sequence of positive numbers $\left\{d_{\varepsilon}\right\}_{\varepsilon}$, as $\varepsilon \rightarrow 0$, and sequence of functions $\left\{\widetilde{u}_{\varepsilon}\right\}_{\varepsilon}$, with $\left(\widetilde{u_{\varepsilon}}, \widetilde{u_{\varepsilon}}\right)_{\varepsilon}=1, \widetilde{u}_{\varepsilon}$ being a linear combination of eigenfunctions of (2.6) associated with all the eigenvalues $\lambda_{\varepsilon, l(\varepsilon)}, \cdots, \lambda_{\varepsilon, l(\varepsilon)+\nu(\varepsilon)}, l(\varepsilon) \in \mathbb{N}, \nu(\varepsilon) \in \mathbb{N}_{0}$, satisfying

$$
\varepsilon^{2} \lambda_{\varepsilon, l(\varepsilon)}, \cdots, \varepsilon^{2} \lambda_{\varepsilon, l(\varepsilon)+\nu(\varepsilon)} \in\left[\lambda-d_{\varepsilon}, \lambda+d_{\varepsilon}\right]
$$

such that $\left\{\tilde{u}_{\varepsilon}\right\}_{\varepsilon}$ converges, as $\varepsilon \rightarrow 0$, to some function $u \neq 0$ in the weak topology of $V^{a} \times V^{b}$, where $V^{a}$ and $V^{b}$ are defined by (3.5). Moreover,

- if $\lambda=\lambda^{a}$ is an eigenvalue of (1.3) and $z^{a}$ is an eigenfunction associated with $\lambda^{a}$, satisfying $\left\|z^{a}\right\|_{L^{2}(\omega)}=1$, and $\mu$ is an eigenvalue of (1.6) and $\alpha$ is an eigenfunction associated with $\mu$, satisfying $\|\alpha\|_{L^{2}(] 0, d[)}=1$, then $\widetilde{u_{\varepsilon}}=\left(\widetilde{u_{\varepsilon}^{a}}, \widetilde{u_{\varepsilon}^{b}}\right)$ can be constructed such that $\left\{\widetilde{u_{\varepsilon}^{a}}\right\}_{\varepsilon}$ converges to $u^{a}=\left(1+\lambda^{a}\right)^{-\frac{1}{2}} z^{a}\left(x_{1}\right) \alpha\left(x_{2}\right)$ in $V^{a}$.

- if $\lambda=\lambda^{b}$ is an eigenvalue of (1.4) and $z^{b}$ is an eigenfunction associated with $\lambda^{b}$, satisfying $\left\|z^{b}\right\|_{L^{2}(]-1,0[)}=1$, and $\mu$ is an eigenvalue of (1.7) and $\beta$ is an eigenfunction associated with $\mu$, satisfying $\|\beta\|_{L^{2}(\omega)}=1$, then the sequence $\widetilde{u_{\varepsilon}}=\left(\widetilde{u_{\varepsilon}^{a}}, \widetilde{u_{\varepsilon}^{b}}\right)$ can be constructed such that $\left\{\widetilde{u_{\varepsilon}^{b}}\right\}_{\varepsilon}$ converges towards $u^{b}=(1+$ $\left.\lambda^{b}\right)^{-\frac{1}{2}} z^{b}\left(x_{2}\right) \beta\left(x_{1}\right)$ in $V^{b}$.

Proof. The proof of item 1 has been performed in Proposition 3.2, see also Remark 3.3.

The proof of the right hand side of the equivalence of item 2 , is also obtained by Theorem 5.2 and Theorem 5.3, in the case where $\lambda$ is an eigenvalue of (1.3) or of (1.4). The rest of the statements follows from Theorem 5.2, Theorem 5.3, and Theorem 5.4.

As regards the proof of the left hand side of the equivalence we follow the idea in Proposition 3.4 with minor modifications. Namely, we proceed by contradiction and we assume that $\lambda$ is not an eigenvalue of (3.7). On account of $\widetilde{u}_{\varepsilon}=\sum_{j=0}^{\nu(\varepsilon)} \beta_{j}^{\varepsilon} u_{\varepsilon, l(\varepsilon)+j}$ for 
certain constants $\beta_{j}^{\varepsilon}$ and $u_{\varepsilon, k}=\left(u_{\varepsilon, k}^{a}, u_{\varepsilon, k}^{b}\right)$ eigenfunctions corresponding to $\lambda_{\varepsilon, k}$ such that $\left\|u_{\varepsilon, k}^{a}\right\|_{L^{2}\left(\Omega^{a}\right)}^{2}+\left\|u_{\varepsilon, k}^{b}\right\|_{L^{2}\left(\Omega^{b}\right)}^{2}=1, k=l(\varepsilon)+j$, for $j=0, \ldots, \nu(\varepsilon)$, we obtain the relation

$$
\begin{aligned}
& \int_{\Omega^{a}}\left(\partial_{x_{1}} \widetilde{u_{\varepsilon}^{a}} \partial_{x_{1}} v^{a}+\varepsilon^{2} \partial_{x_{2}} \widetilde{u_{\varepsilon}^{a}} \partial_{x_{2}} v^{a}\right) d x+\int_{\Omega^{b}}\left(\varepsilon^{2} \partial_{x_{1}} \widetilde{u_{\varepsilon}^{b}} \partial_{x_{1}} v^{b}+\partial_{x_{2}} \widetilde{u_{\varepsilon}^{b}} \partial_{x_{2}} v^{b}\right) d x \\
& =\sum_{j=0}^{\nu(\varepsilon)}\left(\varepsilon^{2} \lambda_{\varepsilon, l(\varepsilon)+j}-\lambda\right) \beta_{j}^{\varepsilon}\left(\int_{\Omega^{a}} u_{\varepsilon, l(\varepsilon)+j}^{a} v^{a} d x+\int_{\Omega^{b}} u_{\varepsilon, l(\varepsilon)+j}^{b} v^{b} d x\right) \\
& +\lambda\left(\int_{\Omega^{a}} \widetilde{u_{\varepsilon}^{a}} v^{a} d x+\int_{\Omega^{b}} \widetilde{u_{\varepsilon}^{b}} v^{b} d x\right), \quad \forall\left(v^{a}, v^{b}\right) \in V_{\varepsilon} .
\end{aligned}
$$

Then, considering that $\left|\varepsilon^{2} \lambda_{\varepsilon, l(\varepsilon)+j}-\lambda\right| \leq d_{\varepsilon}$, we take limits in (5.20) with $\left(v^{a}, v^{b}\right)=$ $\left(\left.v_{\varepsilon}\right|_{\Omega^{a}},\left.v_{\varepsilon}\right|_{\Omega^{b}}\right)$, where $v_{\varepsilon}$ is defined in (3.9), to get that $(\lambda, u)$ satisfies (3.7) and, consequently, $u \equiv 0$, which contradicts our assumption.

\section{Acknowledgments}

This work has partially been supported by MINECO grant MTM2013-44883-P and MICINN grant PGC2018-098178-B-I00. The first author is also member of the Gruppo Nazionale per l'Analisi Matematica, la Probabilità e le loro Applicazioni (GNAMPA) of the Istituto Nazionale di Alta Matematica (INdAM).

\section{References}

[1] D. Borisov and P. Freitas, Singular asymptotic expansions for Dirichlet eigenvalues and eigenfunctions of the Laplacian on thin planar domains, Ann. Inst. H. Poincaré Anal. Non Linéaire 26 (2009), 547-560.

[2] D. Borisov and P. Freitas, Asymptotics of Dirichlet eigenvalues and eigenfunctions of the Laplacian on thin domains in $\mathbb{R}^{d}$, J. Funct. Anal. 258 (2010), 893-912.

[3] G. Bouchitté, M.L. Mascarenhas, and L. Trabucho, On the curvature and torsion effects in one dimensional waveguides, Control Optim. Calc. Var. 13 (2007), 793808.

[4] R. Bunoiu, A. Gaudiello, and A. Leopardi, Asymptotic analysis of a Bingham fluid in a thin T-like shaped structure, J. Math Pures Appl. 123 (2019), 148-166.

[5] G. Cardone, S.A. Nazarov, and K.M. Ruotsalainen, Asymptotics of an eigenvalue in the continuous spectrum of a narrowing waveguide. Mat. Sb. 203 (2012), 3-32; translation in Sb. Math. 203 (2012), 153-182.

[6] C. Castro and E. Zuazua, Une remarque sur l'analyse asymptotique spectrale en homogénéisation, C.R. Acad. Sci. Paris Sér. I 322 (1996), 1043-1047. 
[7] P.G. Ciarlet and P. Destuynder, A justification of the two-dimensional linear plate model, J. Mécanique 18 (1979), 315-344.

[8] P.G. Ciarlet and S. Kesavan, Two-dimensional approximation of three-dimensional eigenvalue problems in plate theory, Comput. Methods Appl. Mech. Engrg. 26 (1981), 149-172.

[9] D. Cioranescu and J. Saint Jean Paulin, Homogenization of Reticulated Structures (Applied Mathematical Sciences). Springer-Verlag, New York, 1999.

[10] L. Friedlander and M. Solomyak, On the spectrum of the Dirichlet Laplacian in a narrow infinite strip, Amer. Math. Soc. Transl. 225 (2008) 103-116.

[11] L. Friedlander and M. Solomyak, On the spectrum of the Dirichlet Laplacian in a narrow strip, Israel J. of Math. 170 (2009), 337-354.

[12] A. Gaudiello, B. Gustafsson, C. Lefter, and J. Mossino, Asymptotic analysis of a class of minimization problems in a thin multidomain, Calc. Var. Partial Differ. Equ. 15 (2002), 181-201.

[13] A. Gaudiello, B. Gustafsson, C. Lefter, and J. Mossino, Asymptotic analysis for monotone quasilinear problems in thin multidomains, Differential Integral Equations 15 (2002), 623-640.

[14] A. Gaudiello and R. Hadiji, Ferromagnetic thin multi-structures, J. Differential Equations 257 (2014), 1591-1622.

[15] A. Gaudiello and A. G. Kolpakov, Influence of non degenerated joint on the global and local behavior of joined rods, Int. J. Eng. Sci. 49 (2011), 295-309

[16] A. Gaudiello, G. Panasenko, and A. Piatnitski, Asymptotic analysis and domain decomposition for a biharmonic problem in a thin multi-structure, Commun. Contemp. Math. 18 (2016), 5, 1550057 (27 pages).

[17] A. Gaudiello and A. Sili, Asymptotic analysis of the eigenvalues of a Laplacian problem in a thin multidomain, Indiana Univ. Math. J. 56 (2007), 1675-1710.

[18] A. Gaudiello and A. Sili, Asymptotic analysis of the eigenvalues of an elliptic problem in an anisotropic thin multidomain, Proc. Roy. Soc. Edinburgh Sect. A 141 (2011), 739-754.

[19] A. Gaudiello and E. Zappale, A model of joined beams as limit of a $2 D$ plate, $J$. Elasticity 103 (2011), 205-233.

[20] Yu.D. Golovaty, D. Gómez, M. Lobo, and E. Pérez, On vibrating membranes with very heavy thin inclusions, Math. Models Methods Appl. Sci. 14 (2004), 987-1034.

[21] D. Gómez, M. Lobo, and E. Pérez, On the eigenfunctions associated with the high frequencies in systems with a concentrated mass, J. Math. Pures Appl. 78 (1999), 841-865. 
[22] D. Gómez, S.A. Nazarov, E. Pérez, Spectral stiff problems in domains surrounded by thin stiff and heavy bands: local effects for eigenfunctions, Netw. Heterog. Media 6 (2011), 1-35.

[23] H. Irago, N. Kerdid and J.M. Viaño, Analyse asymptotique des modes de hautes fréquences dans les poutres minces, C.R. Acad. Sci. Paris Sér. I Math. 326 (1998), 1255-1260.

[24] H. Irago and J.M. Viaño, Asymptotic analysis of torsional and stretching modes of thin rods, Quart. Appl. Math. 58 (2000), 495-510.

[25] I.V. Kamotskii and S.A. Nazarov, On eigenfunctions localized in a neighborhood of the lateral surface of a thin domain, J. Math. Sci., 101 (2000), 2941-2974.

[26] A.M. Khludnev, T.S. Popova, Junction problem for Euler-Bernoulli and Timoshenko elastic inclusions in elastic bodies, Quart. Appl. Math. 74 (2016), 705-718.

[27] A. Klevtsovskiy, T. Mel'nyk Asymptotic approximation for the solution to a semilinear parabolic problem in a thin star-shaped junction, Math. Methods Appl. Sci. 41 (2018), 1, 159-191.

[28] A. Klevtsovskiy, T.A. Mel'nyk, Asymptotic approximations of the solution to a boundary value problem in a thin aneurysm type domain, J. Math. Sci. (N. Y.) 224 (2017), 667-693.

[29] D. Krejčiř́k, Spectrum of the Laplacian in a narrow curved strip with combined Dirichlet and Neumann boundary conditions, ESAIM: Control, Optim. Calc. Var. 15 (2009), 555-568.

[30] H. Le Dret, Problèmes variationnels dans les multi-domaines: modélisation des jonctions et applications. Research in Applied Mathematics, 19. Masson, Paris, 1991.

[31] M. Lobo and E. Pérez, High frequency vibrations in a stiff problem, Math. Models Methods Appl. Sci. 7 (1997), 291-311.

[32] M. Lobo and E. Pérez, The skin effect in vibrating systems with many concentrated masses, Math. Methods Appl. Sci., 24 (2001), 59-80.

[33] M. Lobo and E. Pérez, Local problems for vibrating systems with concentrated masses: a review. C. R. Mécanique 331 (2003), 303-317.

[34] M. Lobo and E. Pérez, Long time approximations for solutions of wave equations associated with the Steklov spectral homogenization problems. Math. Methods Appl. Sci., 33 (2010), 1356-1371.

[35] S.A. Nazarov, On the asymptotics of the spectrum of a problem in elasticity theory for a thin plate. Sibirsk. Mat. Zh. 41 (2000), 895-912; translation in Siberian Math. J. 41 (2000), 744-759. 
[36] S.A. Nazarov, E. Pérez, and J. Taskinen, Localization effect for Dirichlet eigenfunctions in thin non-smooth domains, Trans. Amer. Math. Soc. 368 (2016), 4787-4829.

[37] S.A. Nazarov and A.S. Slutskij, Asymptotic analysis of an L-shaped junction, J. Math. Sci. (N.Y.) 216 (2016), 279-312.

[38] O.A. Oleinik, A.S. Shamaev, and G.A Yosifian, Mathematical Problems in Elasticity and Homogenization. North-Holland, London, 1992.

[39] G.P. Panasenko and E. Pérez, Asymptotic partial decomposition of domain for spectral problems in rod structures, J. Math. Pures Appl. 87 (2007), 1-36.

[40] E. Pérez, On the whispering gallery modes on interfaces of membranes composed of two materials with very different densities. Math. Models Methods Appl. Sci. 13 (2003) 75-98.

[41] E. Pérez, Long time approximations for solutions of wave equations via standing waves from quasimodes. J. Math. Pures Appl. 90 (2008), 387-411.

[42] J. Sanchez-Hubert and E. Sanchez-Palencia, Vibration and Coupling of Continuous Systems. Asymptotic Methods. Springer-Verlag, Heidelberg, 1989. 